\title{
Analysis of Various Algorithmic approaches to Software-Based 1200 Baud Audio Frequency Shift Keying Demodulation for APRS
}

\section{A Thesis}

\section{Presented to}

the Faculty of California Polytechnic State University,

San Luis Obispo

\author{
In Partial Fulfillment \\ of the Requirements for the Degree \\ Master of Science in Computer Science
}

by

Robert F. Campbell

June 2016 
(C) 2016

Robert F. Campbell

ALL RIGHTS RESERVED 


\section{COMMITTEE MEMBERSHIP}

TITLE:

AUTHOR:

DATE SUBMITTED: June 2016

COMMITTEE CHAIR: John Bellardo, Ph.D. Associate Professor, Computer Science

COMMITTEE MEMBER: Bridget Benson, Ph.D. Assistant Professor, Electrical Engineering

COMMITTEE MEMBER: Dennis Derickson, Ph.D.

Department Chair, Electrical Engineering 


\begin{abstract}
Analysis of Various Algorithmic approaches to Software-Based 1200 Baud Audio Frequency Shift Keying Demodulation for APRS
\end{abstract}

\title{
Robert F. Campbell
}

Digital communications continues to be a relevant field of study as new technologies appear and old methodologies get revisited or renovated. The goal of this research is to look into the old digital communication scheme of Bell $202[67]$ used by APRS and improve software based demodulation performance. Improved performance is defined by being able to correctly decode more packets in an efficient, real time, manner. Most APRS demodulation is currently done using specialized hardware since that yields the best performance. This research shows that through using Sivan Toledo's javAX25 [72] software package, new demodulation algorithms can be implemented that decode more Bell 202 encoded AX.25 packets than the existing software could. These improvements may help drive the adoption of software demodulation since it is a low cost alternative to specialized hardware. 


\section{TABLE OF CONTENTS}

LIST OF TABLES . . . . . . . . . . . . . . . . . . . . vii

LIST OF FIGURES $\ldots \ldots \ldots \ldots \ldots \ldots \ldots \ldots \ldots$ viii

\section{CHAPTER}

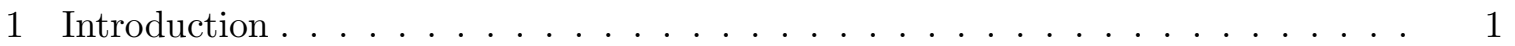

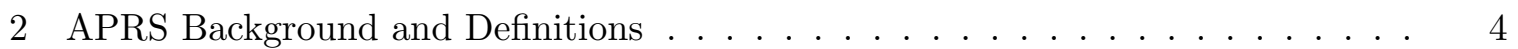

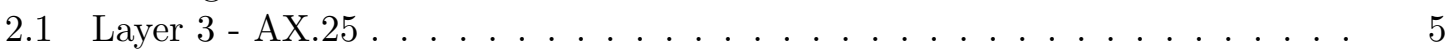

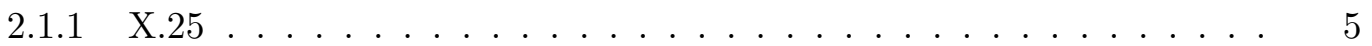

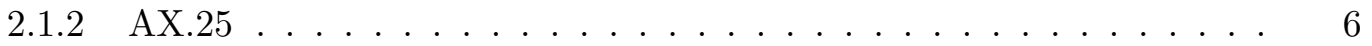

2.2 Layer 2 - High-Level Data Link Control _ . . . . . . . . . . . . . 6

2.3 Layer 1 - Bell 202 Modulation and the Radio . . . . . . . . . . . . . 7

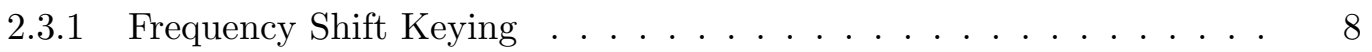

$2.3 .2 \quad$ Bell $202 \ldots \ldots \ldots \ldots \ldots \ldots$

3 Approaches to Accessing the APRS Network _ . . . . . . . . . . . 10

3.1 Terminal Node Controllers . . . . . . . . . . . . . . . . . . . . . 10

3.2 Specialized APRS Hardware . . . . . . . . . . . . . . . . . . . . . 12

3.3 Software Based Demodulation . . . . . . . . . . . . . . . . . . . . 13

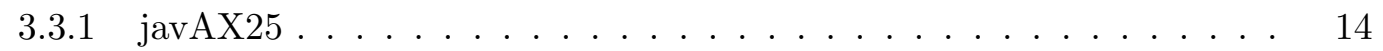

4 Bell 202 Demodulation Techniques . . . . . . . . . . . . . . . . 16

4.1 Edge Detection . . . . . . . . . . . . . . . . . . . . . 18

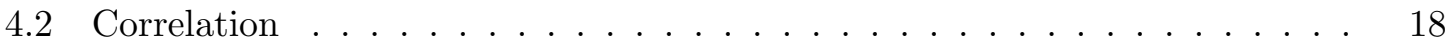

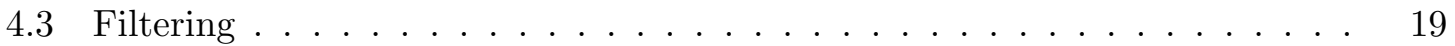

4.3 .1 Discrete Fourier Transform . . . . . . . . . . . . . . . 20

4.3 .2 Goertzel Algorithm . . . . . . . . . . . . . . . . . . . . 20

4.4 Phase Locked Loop . . . . . . . . . . . . . . . . . . . . . . . 21

4.5 Additional Benefits of Software Based Decoding . . . . . . . . . . . . . 21

4.5.1 Exhaustive Search of Incoming Signal . . . . . . . . . . . . . . 23

4.5.2 Taking Advantage of Parallel Demodulation . . . . . . . . . . . . 23

5 Demodulation Challenges . . . . . . . . . . . . . . . . . . . . . . . . 24

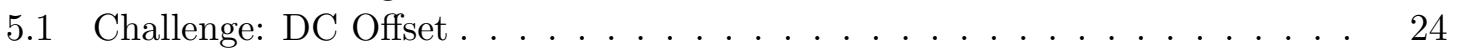

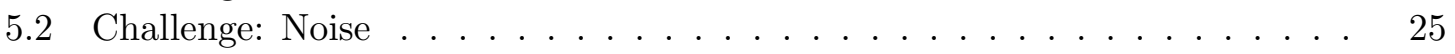

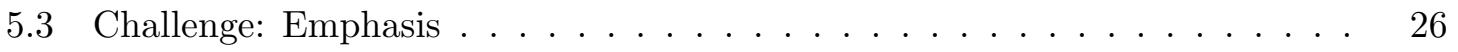

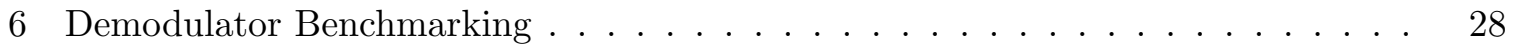

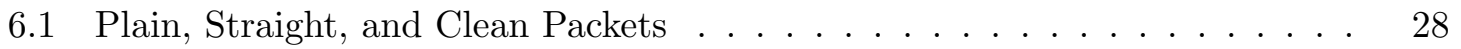

6.2 White Noise Testing . . . . . . . . . . . . . . . . . . . . . . . . . . 29

6.3 Los Angeles APRS Test Recordings . . . . . . . . . . . . . . . . 30

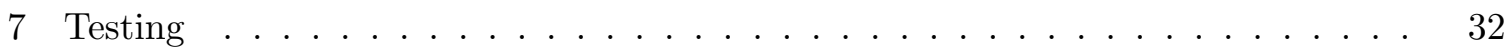

7.1 Hardware Testing Setup . . . . . . . . . . . . . . . . . . . 32

7.1.1 Setting TNC Audio Levels . . . . . . . . . . . . . . . . 32

7.2 Software Testing Setup . . . . . . . . . . . . . . . . . . 34

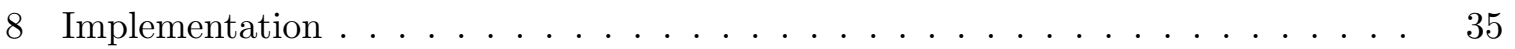

8.1 Strict Zero Crossing Demodulator _ . . . . . . . . . . . . . . 35

8.2 Floating Ground Zero Crossing Demodulator . . . . . . . . . . . . 35

8.3 Windowed Zero Crossing Counting Demodulator . . . . . . . . . 36 
8.4 Peak Detection Demodulator . . . . . . . . . . . . . . . 36

8.5 Derivative Zero Crossing Demodulator . . . . . . . . . . . . . 37

8.6 Goertzel Filter Demodulator _. . . . . . . . . . . . . . 37

8.7 Phase Locked Loop Demodulator . . . . . . . . . . . . . . . . . . . . 38

8.8 Mixed Preclocking Demodulator . . . . . . . . . . . . . . . . . 38

8.9 Goertzel Preclocking Demodulator . . . . . . . . . . . . . . . 39

8.10 Goertzel Exhaustive Preclocking Demodulator . . . . . . . . . . . 39

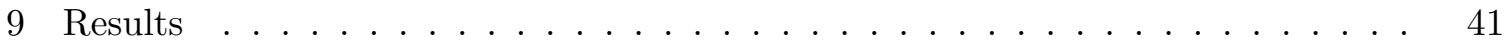

9.1 Dedicated Hardware Results . . . . . . . . . . . . . . . . . . . . 41

9.2 Software Results . . . . . . . . . . . . . . . . . . . . 43

9.2.1 Hardware and Software Comparisons . . . . . . . . . . . . . . . 49

10 Future Work . . . . . . . . . . . . . . . . . . . 56

10.1 The Discrete Short-Time Fourier Transform . . . . . . . . . . . . . 56

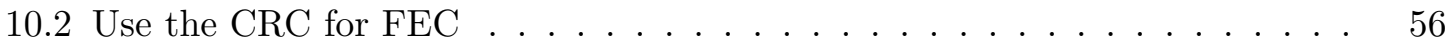

10.3 Integrations with a Radio . . . . . . . . . . . . . . 57

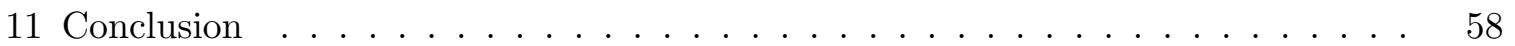

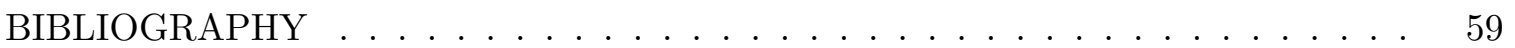




\section{LIST OF TABLES}

Table Page

1.1 Cost comparison of conducting APRS communications on 1 through 4 channels for hardware versus software. . . . . . . . . . . . . . .

2.1 Example packet showing the results of bit stuffing and framing with leading and trailing flags. . . . . . . . . . . . . . . . 7

4.1 Hardware Demodulation Techniques $[31,18,16,33,43,2] \ldots \ldots \ldots$

7.1 Minimum Optimal TNC Input Audio Levels $[35,43,2,31] \ldots \ldots$. . . . . . . 34 


\section{LIST OF FIGURES}

Figure

1.1 Block diagram of an example APRS system end to end. . . . . . . . . . .

2.1 Example Bell 202 signal encoding the bit stream '0000'. The bit is determined at the bit period boundary with a change in frequency representing a ' 0 ' and no change in frequency representing a '1'. Since a frequency transition occurs at every visible boundary in this plot (at samples 40, 80, 120, and 160) the underlying data is '0000'. Without knowing the frequency before sample 0 or after sample 200 no other bits can be determined. . . . . . . . . . . . .

3.1 Image of the Kantronics KAM Plus TNC [37] . . . . . . . . . . . . . . .

3.2 Image of Argent Data's OpenTracker $3[15] \ldots$. . . . . . . . . . . . . . . . Image of the Yaesu FTM-350 Radio which has APRS integrated [83]. . . . . Example of a coherent FSK signal. . . . . . . . . . . . . . . Example of a non-coherent FSK signal. Block diagrams for (a) A Correlation Receiver and (b) A Matched Filter Receiver [14].

Block Diagram of a time delay correlator [58] Block diagram of a PLL demodulator [51]. Circuit connection for FSK Decoding of Bell 202 Format using an Exar XR-

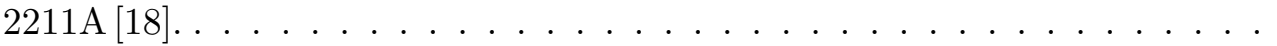

5.1 An example Bell 202 signal with DC offset problems. . . . . . . . . . . .

5.2 An example Bell 202 signal with white noise added. The additional zero crossings at sample 9 in the first bit period and sample 169 in the last bit period will make these $1200 \mathrm{~Hz}$ bit periods look more like $2200 \mathrm{~Hz}$ signals. . .

5.3 An example signal that was not preemphasized, but was deemphasized. . .

6.1 Example of the AFSK signal present in the 200 packet generated file. . . . .

6.2 Example of a generated AFSK signal with artificial white noise added. . . .

6.3 Example of the AFSK signal in Track 1 of the Los Angeles Recording Test File. . . . . . . . . . . . . . . . . . . . . .

7.1 Example Radio Port pin out for Kantronics, also consistent with others including OpenTrackers [41]. . . . . . . . . . . . . . . . . 33

7.2 Break-out board fabricated for hardware testing. . . . . . . . . . . . . 33

7.3 Block diagram of the test setup used for testing APRS hardware. . . . . . .

9.1 Number of packets successfully decoded for all tested hardware on the OpenTracker 3 test file with noise. . . . . . . . . . . . . . . .

9.2 Number of packets successfully decoded for all tested hardware on the Track 1 test file. . . . . . . . . . . . . . . . . . . . .

9.3 Number of packets successfully decoded for all tested hardware on the Track 2 test file. . . . . . . . . . . . . . . . . . . . . 44

9.4 Performance of software on the raw signal from OpenTracker 3 Test. . . . . 45

9.5 Performance of software on OpenTracker 3 Test with a flat bandpass filter. $\quad 46$

9.6 Performance of software on OpenTracker 3 Test with an emphasis filter. . . 46

9.7 Performance of Software on the raw signal from Generated 200. . . . . . . . 47

9.8 Performance of software on Generated 200 with a flat bandpass filter. . . . 47

6

47


9.9 Performance of Software on Generated 200 with an emphasis filter. . . . . .

9.10 Performance of software on the raw signal from OpenTracker Test with noise

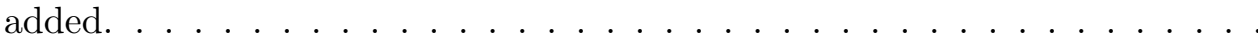

9.11 Performance of software on OpenTracker Test with noise added with a flat bandpass filter. . . . . . . . . . . . . . . . . .

9.12 Performance of Software on OpenTracker Test with noise added with an emphasis filter. . . . . . . . . . . . . . . 51

9.13 Performance of software on the raw signal from Track 1. . . . . . . . . 51

9.14 Performance of software on Track 1 with a flat bandpass filter. . . . . . . . 52

9.15 Performance of software on Track 1 with an emphasis filter. . . . . . . . . . 52

9.16 Performance of software on the raw signal from Track 2. . . . . . . . . 53

9.17 Performance of software on Track 2 with a flat bandpass filter. . . . . . . . 53

9.18 Performance of software on Track 2 with an emphasis filter. . . . . . . . 54

9.19 Performance of software versus hardware on Track 1 . . . . . . . . . . 54 


\section{Introduction}

Amateur Radio Operators, commonly referred to as "hams," make the best of resources available to them. However, once something is working a "don't touch it if it ain't broke" approach is often taken. Between these two mentalities some interesting phenomenon have occurred within the ham community. For example, some radio systems that are in active service today have only seen very minimal attention since the 1980's when they were originally installed. The implementation and development of the Automated Packet Reporting System (APRS) is no exception to the way hams approach things [6]. Much of this system is based off older hardware and protocols - from the 1980s - that were readily available and few improvements have been made. Unfortunately, although the specification has been relatively stable there are inconsistencies. These inconsistencies include varying implementations from vendor to vendor as well as portions of the specification that are not clearly defined resulting in vastly inconsistent performance $[19,20]$.

So, what is APRS, and why does it matter? A brief introduction to APRS is that it is a digital communication scheme used by hams where a packet (whose content is varied, but is usually a GPS position - which is what gave APRS it's original name "Automated Position Reporting System" [76]) is sent out over radio and then interpreted by other receiving stations. Figure 1.1 shows one example of an end to end APRS system which starts with The GPS communicating information using NEMA to the APRS tracker, the tracker then takes this information and the preferences in its configuration to formulate APRS packets. The APRS packets are encoded by the tracker and the resulting audio passed to a transmit radio which sends the data. Typically this is done on frequency $144.390 \mathrm{MHz}$ in the United States. Receiving radios tuned to the same frequency pass the received audio to a TNC which decodes the packet and passes it to a computer using RS-232.

A major challenge to this protocol and many other methods of digital communication is the fact that it uses radio. Transmitting the signal wirelessly over radio means that it is susceptible to interference, weak signals as the distance from the transmitting station increases, as well as a myriad of other items. This research focuses specifically on the receiving end of these signals in order to see what improvements can be made to software based approaches to demodulating (decoding) these packets, which is represented by the TNC block in Figure 1.1. However, to more accurately portray software based demodulation 


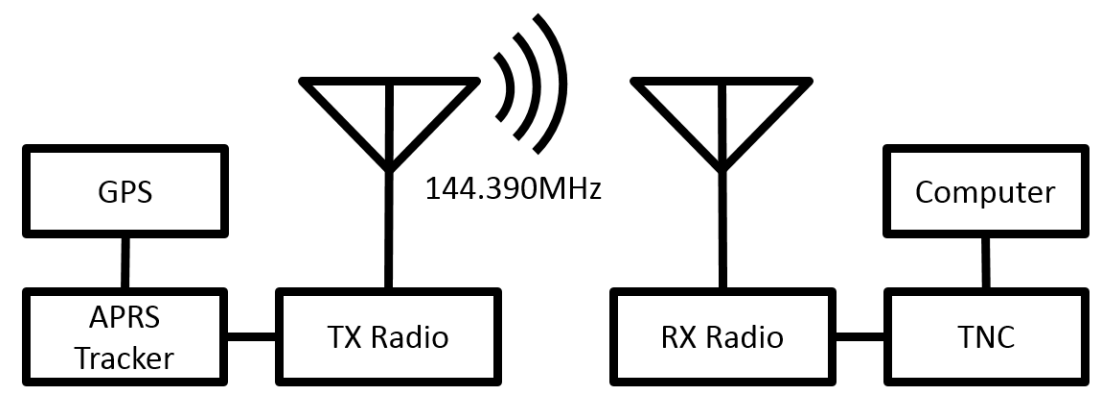

Figure 1.1. Block diagram of an example APRS system end to end.

the TNC block in the figure should be moved inside of the computer block since the received radio audio is passed straight to the computer and then interpreted by this special software.

The reasoning for trying to make improvements in software based demodulation are many, but a few of the more motivational ones are to follow. One advantage of doing software based demodulation is that it removes the necessity of specialty hardware; Instead of having dedicated hardware whose sole purpose is to modulate and demodulate APRS packets, hams can use a computer to do these tasks. By using a computer's sound card, audio from the radio can be processed using software, which will decode received packets, or audio can be played from the sound card to the radio to be transmitted. With the abundance of personal computers, this can provide a much cheaper solution for hams who are interested in trying out APRS without having to put down a potentially big initial investment $(\sim \$ 200[36,47])$ for a piece of hardware that serves one purpose. The price of this specialty hardware is steep and it is limited to only performing communication on a single channel. When using a line in / out on a computer they are typically stereo, meaning that a single sound card could handle operations on multiple channels. If two channels just is not enough the capabilities of a computer demodulator can be expanded by merely adding another sound card which is relatively cheap at $\sim 20[46]$. See Table 1.1 for a comparison of the cost for hardware and software. From the table it can be seen that the cost to perform communication on 4 channels using dedicated hardware the cost would be $\$ 800$ ! For this cost a whole computer with a half dozen sound cards could be purchased, only further expanding capabilities.

In addition to the the price advantages of software based demodulation approaches, there is also one other primary advantage. If software is being used instead of hardware there is the 


\section{CHAPTER 1. INTRODUCTION}

Table 1.1: Cost comparison of conducting APRS communications on 1 through 4 channels for hardware versus software.

\begin{tabular}{|l|r|r|r|r|}
\hline Cost for: & 1 Channel & 2 Channels & 3 Channels & 4 Channels \\
\hline Software & $\$ 0$ & $\$ 0$ & $\$ 20$ & $\$ 20$ \\
\hline Hardware & $\$ 200$ & $\$ 400$ & $\$ 600$ & $\$ 800$ \\
\hline
\end{tabular}

potential for a lot more capabilities since processing power and available memory increase drastically. For instance, one of the dedicated hardware solutions, the Kantronics KPC-3 Plus, has a mere $512 \mathrm{~KB}$ of memory compared to that of any computer which is over $4 \mathrm{~GB}$ as of 2014 - and that is just the ram, not hard drive space [36, 25]. Additionally, instead of just being able to handle live events and process each data point in the best manner possible as soon as it comes in, post processing becomes an option.

With the cost and versatility of a software demodulation solution now introduced, the paper addresses the following: Chapter 2 goes into background information, with a deeper introduction to APRS and a presentation of the aspects important to understanding this research. In Chapter 3, some of the current methods for interfacing with APRS, both hardware and software, are explained. Demodulation techniques are discussed in Chapter 4. Chapter 5 talks about the challenges of demodulating APRS packets. Chapter 6 discusses the methods used for benchmarking and comparing the demodulators. In Chapter 7, information on how the demodulators and algorithms are tested is presented. Chapter 8 goes into more detail about the software implementations in this project. Chapter 9 discusses the results of both the newly implemented algorithms and compares them to other demodulators. Areas of additional research and future work are discussed in Chapter 10. Chapter 11 is concluding remarks. 


\section{APRS Background and Definitions}

Thus far APRS has been introduced as a method of digital communication used by hams in order to inform other hams of their location. In addition to supporting sending positions, APRS can be used to send messages, bulletins, weather, and other information. Since these packets are transmitted via radio - which has limited coverage - APRS should be viewed as a local area awareness network. This gives hams who are listening for and decoding APRS packets information about nearby transmitting stations. These previous few sentences give a brief overview of what APRS is from a user's perspective, but the rest of the section will focus more on what is going on behind-the-scenes to explain how APRS works in terms of the protocols, data transmission, modulation, etc. The full specification (version 1.0.1) published in 2000 can be found at reference [27] and the 1.1 and proposed 1.2 addendum at $[7,8]$, for those interested. Although the APRS specification was published in 2000, APRS over VHF has the 1980s technology of Bell 202 at its heart. It is worth pointing out that depending on where one looks, APRS may be an acronym for either Automatic Packet Reporting Service [6], Automatic Position Reporting Service [64], Amateur Packet Reporting Service [29], or others.

This discussion of the different components of APRS will be handled by breaking down APRS into the layers of the Open Systems Interconnection (OSI) model. However, before fitting APRS into the OSI model it is important to keep in mind the relevant layers that are going to be discussed: Layer 1 of the OSI model is the physical layer, which consists of everything that is used to transport one bit of information from one location to another. The second layer is the data link layer. Within the data link layer bits from the physical layer are passed up to the network layer, and information from the network layer is framed and handed off to the physical layer. Layer 3 is the Networking layer, which is responsible for determining the path that packets will take and for providing flow control to prevent flooding. Above these are layers four through seven which are the transport, session, presentation, and application layers respectively [66]. For APRS, these upper layers get too inter-tangled to be able to cleanly separate them. For instance, within the AX.25 2.2 specification a TNC is mentioned that only implements layers 1,2 , and 7 of the OSI model [4].

The best division of APRS into the OSI model is as follows, with a more detailed and individualized discussion after this introduction. Layer 3 of the OSI model for APRS is the 


\section{CHAPTER 2. APRS BACKGROUND AND DEFINITIONS}

the AX.25 Protocol; High-Level Data Link Control (HDLC) protocol composes layer 2. All the way at the bottom, layer 1 for APRS consists of the Terminal Node Controller (TNC), utilizing a variation of Bell 202 modulation, and a Radio[60]. A brief note on why the discussion begins with Layer 3, and does not address Layers 4-7, is because this is how the data is transferred. The interest in terms of this research stops here and does not continue to the layers above layer three, because those are all application specific and do not have a direct influence on decoding the data from the raw bit stream. Starting with AX.25 the background information will be given down to Layer 1 which is where this research actually aims to make a contribution.

\subsection{Layer 3 - AX.25}

Layer 3, the network layer, is responsible for routing frames between individual nodes in the network. A frame of data is more traditionally called a packet since AX.25 is a packet switched network protocol[49]. AX.25 is the amateur X.25 protocol, hence the prefixed letter 'A'. As such, the AX.25 protocol is the ham's interpretation of the X.25 protocol. Since the origins of AX.25 lie within X.25, the discussion will begin with X.25.

\subsubsection{X.25}

Developed in the 1970's, the packet switching protocol X.25 was deployed on telephone networks where it was used until it began to be displaced by the IP protocol. The X.25 protocol suite provides OSI layers 1-3, although it does have standards that support each of those layers [66]. For instance the X.21 standard was commonly used for layer 1 of X.25 and ISO 7776 specifies a Link Access Procedure Balanced (LAPB) to assist with layer 2 (the data link layer) [22]. The data link layer of LAPB, a bit oriented protocol derived from HDLC, manages packet framing and ensures that frames are error-free and properly sequenced. When used on telephone networks there are five distinct modes that the protocol operates in: call setup for establishing the connection, data transfer, idle where the connection is established but no data is being transferred, call clearing for terminating the connection, and restart for resynchronizing the host and client [34]. Some of the features of the Layer 2 and Layer 3 operations of X.25 can be found in a similar fashion in AX.25. 


\section{CHAPTER 2. APRS BACKGROUND AND DEFINITIONS}

\subsubsection{AX.25}

In this section, the AX.25 protocol will be discussed through comparison and contrast with X.25. One of the main differences between the X.25 and AX.25 is that when the specification is read, in addition to specifying the behavior of Layer 3, the behavior of Layer 2 is specified. Although this is somewhat implied for X.25, there are still separate documents for the specifications for each one of the layers. The specification for AX.25 very clearly defines the framing with starting and terminating flags as well as the networking and routing [4]. This means that one specification and protocol defines two layers in the OSI model. Both X.25 and AX.25 use HDLC derivative for layer two, while AX.25 uses Bell 202 for layer one instead of the X.21 specification used by X.25.

\subsection{Layer 2 - High-Level Data Link Control}

Layer 2 of the OSI model which is responsible for framing the bits, or packets, from layer 3 is taken on by High-Level Data Link Control (HDLC) for APRS. The goal of HDLC is to make sure that when the data is received and passed up to Layer 3, it is error free, without loss, and in the correct order [34]. There are a few ways that HDLC accomplishes this, two of which are framing and the Frame Check Sequence (FCS). The framing occurs through the use of flags around the data. A flag is one byte and is hex 0x7E. For AX.25, common practice is to send multiple flags consecutively to give the transmitting radio time to key up and settle and to give receiving radios time for their squelch (an item that stops radio output when the desired signal strength is too low) to open. Since HDLC is an nonreturn to zero inverted (NRZI) encoding, no change in frequency corresponds to a 1 and a change in frequency corresponds to a 0 . As such multiple 1s in a row make it hard to keep timing, since consecutive 1s are encoded as the same frequency and the timing can only be determined when a frequency change is detected, which is why bit stuffing is used. With the exception of the flag containing six consecutive 1s $(0 \times 7 \mathrm{E}=01111110)$, if there are six or more consecutive $1 \mathrm{~s}$ in the data packet, a zero will be stuffed after the fifth 1 to increase the clocking energy by forcing a frequency transition in the transmitted signal. In addition to increasing the clocking energy, bit stuffing also serves the more important purpose of making sure that there is nothing that can be confused with a flag in the data 


\section{CHAPTER 2. APRS BACKGROUND AND DEFINITIONS}

Table 2.1: Example packet showing the results of bit stuffing and framing with leading and trailing flags.

\begin{tabular}{|l|c|}
\hline Data Packets & {$[11111111][11111111]$} \\
\hline Packet After Bit Stuffing & {$[111110111][1101111101]$} \\
\hline Packet Transmitted Including Flags & {$[01111110][111110111][1101111101][01111110]$} \\
\hline
\end{tabular}

stream; the only place there will be six consecutive 1 symbols is exclusively in the flags that signal the start and end of the packet [30]. See Table 2.1 for and example showing what gets transmitted when the data is two bytes of $1 \mathrm{~s}$, this example data stream will require bit stuffing.

\subsection{Layer 1 - Bell 202 Modulation and the Radio}

Since Layer 1 is composed of all the items needed to transmit one bit of information from one location to another, it needs to be made clear what all is needed for APRS. APRS's layer 1 starts with the air, the medium through which the radio frequency (RF) signals propagate, the $\mathrm{RF}$ transmissions are either received or transmitted by a radio. Then, the audio that the radio receives has to be processed. In order to stay focused on what happens in layer 1 and not start mixing the other layers together, a further discussion of what this audio signal consists of is necessary.

The audio signal that contains the APRS packet is composed using the Bell 202 modulation which is an Audio Frequency Shift Keying (AFSK) mode [1, 24]. As such the complete signal flow takes one of two paths, either incoming or outgoing. Incoming signals start with the $\mathrm{RF}$ coming in through the radio, being translated to the corresponding audio, and then demodulated into a bit stream by interpreting the Bell 202 modulation. Alternatively for outgoing signals, a bit stream from layer 2 of APRS is modulated using the Bell 202 modulation, this modulated audio is passed to the radio, and the radio then transmits it out. Please refer back to Figure ?? for a block diagram. Since decomposing the radio down into its individual components does not have any affect on representing the different OSI layers of APRS, it will not be discussed. However there are factors that affect wireless communications and RF signals that will be discussed in chapter 5 . 


\section{CHAPTER 2. APRS BACKGROUND AND DEFINITIONS}

\subsubsection{Frequency Shift Keying}

There are multiple ways to encode information into a sinusoidal signal. Among these are amplitude modulation (AM), phase modulation (PM), and frequency modulation (FM) [24]. As each one of the names implies, the underlying data is encoded by modifying that corresponding part of the sinusoidal signal - either the amplitude, phase, or frequency [32]. In order to understand the Bell 202 modulation scheme, the communication mode of AFSK needs to be introduced which derives from a FM modulation scheme. AFSK is a form of frequency shift keying (FSK) that occurs by modulating frequencies in the audible range. FSK uses multiple frequencies in order to represent the different symbols such as 1 and 0 or mark and space. If the frequency of the data carrying signal can be determined, then the symbol is known for that bit period. One example of FSK, as opposed to AFSK, is 9600 baud (bits per second) packet radio on Ultra High Frequencies (UHF). In this mode, the actual RF carrier in the $440 \mathrm{MHz}$ band is modulated between one frequency and another nearby frequency in order to represent the two different symbols. In contrast, AFSK switches between two different audio tones, which for APRS on Very High Frequencies (VHF) is then modulated onto the RF carrier using FM.

\subsubsection{Bell 202}

With FSK and AFSK now introduced the AFSK used within Bell 202 can be described. Bell 202 is an older technology with the Bell 202 integrated circuit filter patented in 1984 using $1200 \mathrm{~Hz}$ and $2200 \mathrm{~Hz}$ tones, although the patent was originally filed in 1981 [67]. After the patent was filed it took the International Telecommunication Union (ITU) another 7 years to publish a standard for this modulation in 1988 that was used in telephone networks. In the standard, however they use $1300 \mathrm{~Hz}$ tone for a mark symbol and $2100 \mathrm{~Hz}$ tone for a space symbol [1]. The convention in FSK is for the higher frequency to correspond to the mark and the lower one be the space [74]. An example Bell 202 AFSK signal can be seen in Figure 2.1. In Figure 2.1 and others to follow, the horizontal axis is a relative sample number from the audio file. Since all audio was captured at $48000 \mathrm{~Hz}$ and the Bell 202 signal is 1200 baud this works out to each baud being 40 samples. 
Data Signal Encoding Bit Stream 0000

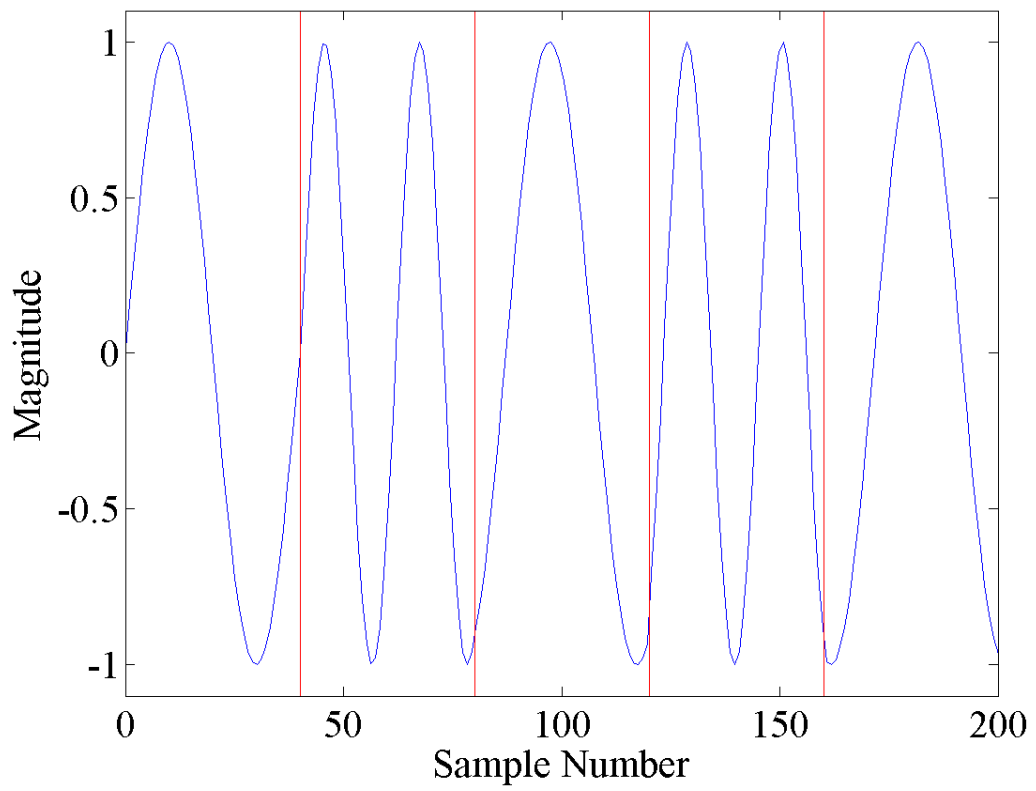

Figure 2.1. Example Bell 202 signal encoding the bit stream '0000'. The bit is determined at the bit period boundary with a change in frequency representing a ' 0 ' and no change in frequency representing a ' 1 '. Since a frequency transition occurs at every visible boundary in this plot (at samples 40, 80, 120, and 160) the underlying data is '0000'. Without knowing the frequency before sample 0 or after sample 200 no other bits can be determined. 


\section{Approaches to Accessing the APRS Network}

In the world of APRS, there are many solutions that hams take advantage of in order to utilize the network. Some they find and make work, some they purchase to use exclusively for APRS, and some go through the trouble of building their own solutions. This chapter explains some of the common systems used on the APRS network, primarily those that can be used for receiving, starting with terminal node controllers and progressing to software based demodulation.

\subsection{Terminal Node Controllers}

Currently there are many systems that will demodulate Bell 202 encoded APRS packets. The original hardware used for this communication style was dedicated modems similar to dial-up 56k modems that did the encoding and decoding. These modems were connected directly to the radio and would decode the signal that the radio received as well as sending audio to the radio to transmit [82]. These modems are more commonly referred to as terminal node controllers (TNCs), which are specialized modems used in APRS operation. A reminder of the age of the technologies that are being used for the data transport of APRS, packet radio originated in the 1980s as TNCs became affordable [28]. This means that this technology is over 30 years old at the time of writing in the year 2016.

With a radio and a $\mathrm{TNC}$, amateur packet stations and digipeaters (digital packet repeaters) are possible $[26,75]$. Digipeaters are an essential part of APRS, but many users wish to report their GPS position onto the network instead of just relaying traffic for other stations. In order to accomplish this, a GPS receiver is required. Now, stations can take the data from their GPS receiver and put it in the payload of the APRS packet and transmit the GPS position onto the network. One other common use of a TNC is an Internet attached digipeater, usually though the use of a computer, that would allow the data to be posted to the APRS-IS servers [12]. To give an example of some of the TNCs available, those within the testing scope of this project will be listed. There are a total of eight TNCs - six unique models - whose decoding results are compared to the software approaches. This includes two Kantronics KAM Plus, a Kantronics KAM, an MFJ-1278, two AEA PK-88, a PK-232, and a PK-232MBX. An example image of what a TNC looks like can be seen in Figure 3.1. 


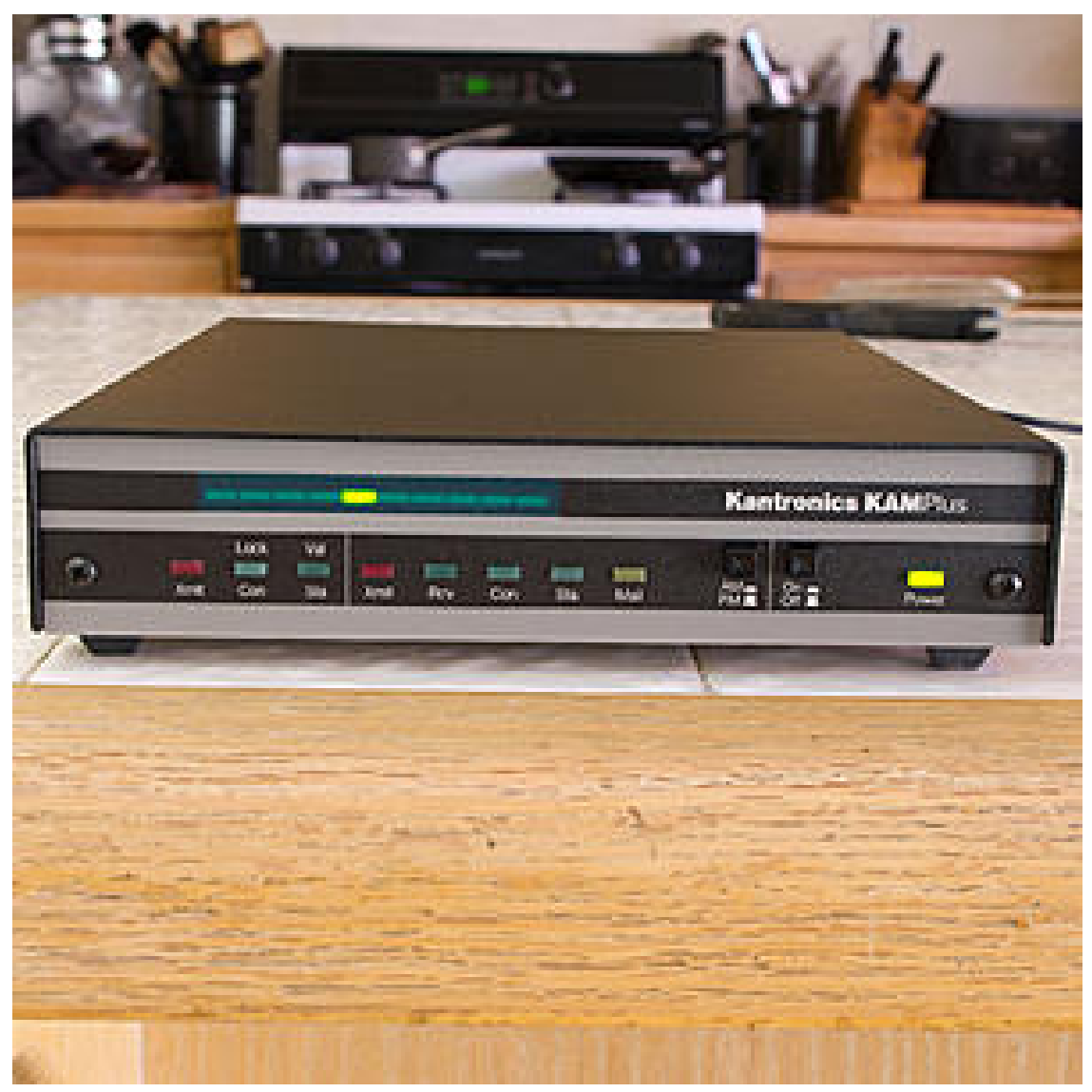

Figure 3.1. Image of the Kantronics KAM Plus TNC [37]. 


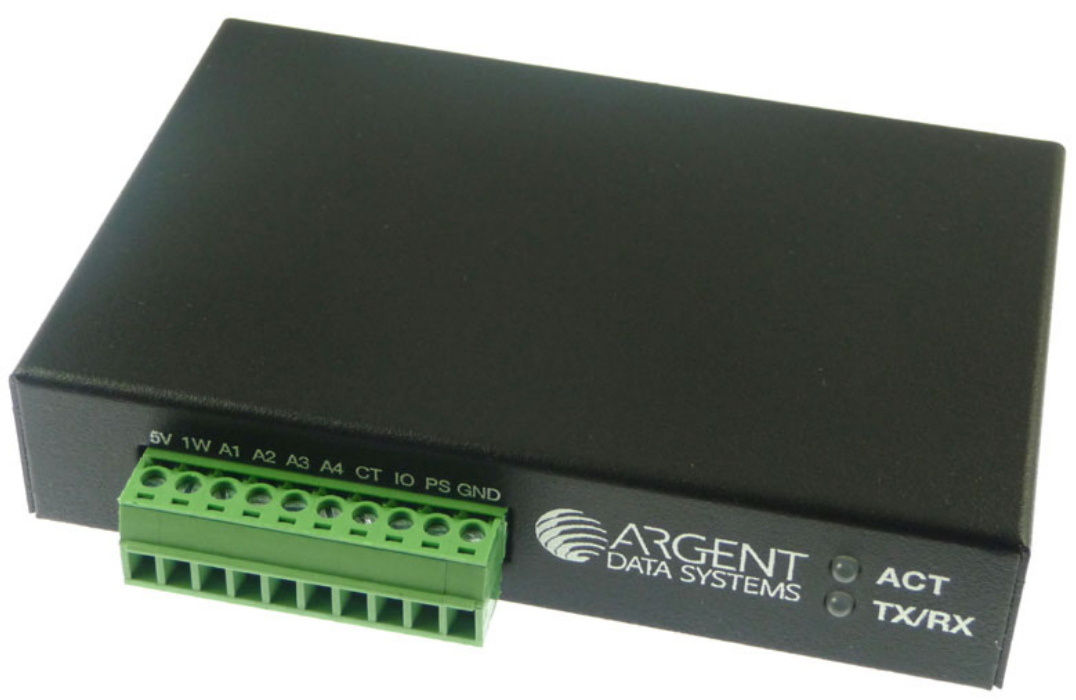

Figure 3.2. Image of Argent Data's OpenTracker 3 [15].

\subsection{Specialized APRS Hardware}

Many hams know exactly what they would like to do with APRS and exactly what traffic they want to contribute to the APRS network. So, instead of purchasing an expensive Multi-mode TNC, companies are making dedicated APRS solutions available to consumers for a fraction of the price. In addition to making this hardware available, the producers support the hardware and make pretty user interfaces for the users to be able to program the hardware exactly as they like and without having to invest much time into understanding how different components work together. Some examples of APRS exclusive devices are Argent Data's OpenTrackers (Figure 3.2), Byonics' TinyTrack, and Fox Delta's Fox Track $[44,9,69]$. These compact packages along with a radio and a GPS module perform APRS tasks at a satisfactory level for many users.

Since the average user only wants to report positional information, these dedicated devices are simple to setup to do such tasks, but include only a simple feature set. Since they are all small embedded systems they cannot perform all of the features that APRS supports, one example is the messaging service. Since these devices do not have a display or a keypad, 


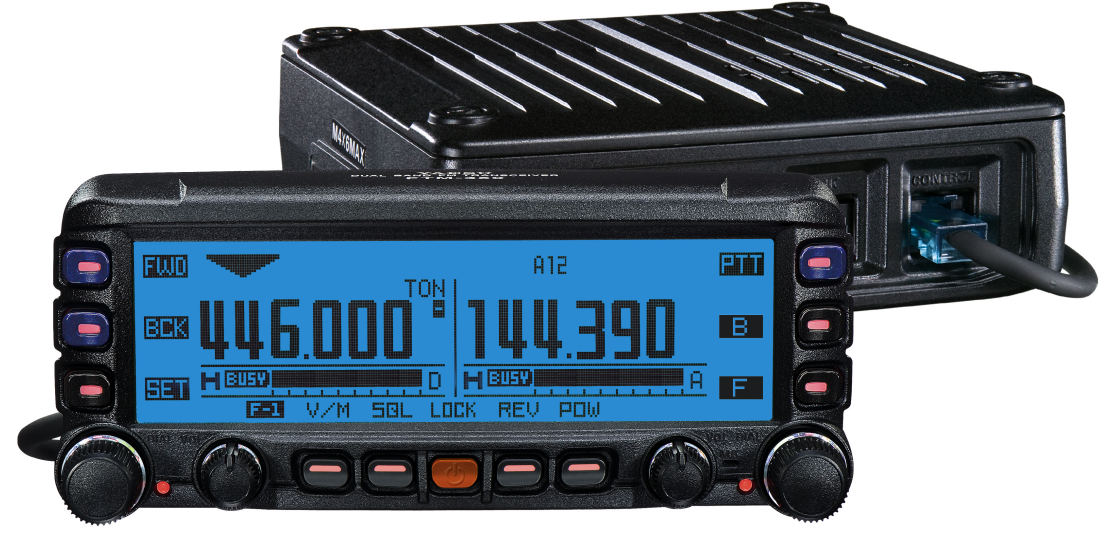

Figure 3.3. Image of the Yaesu FTM-350 Radio which has APRS integrated [83].

there is no way to input or display a message. Certain radio manufacturers have begun integrating the TNCs into the radios themselves to utilize the radio's screen. The Kenwood TM-D700 series and Yaesu FTM-350 (Figure 3.3) are examples [13, 73].

However, both the options in this section and the one previous on TNCs require going out and buying special hardware in order to utilize APRS. This can be expensive and cost prohibitive for some hams to be able to begin APRS operations.

\subsection{Software Based Demodulation}

It's fair to assume that before a ham operator becomes interested in the APRS network and sending APRS packets that they will already have a radio. So, if they already have a radio all they have to do is buy a piece of hardware that will do the modulation in order to send a packet. However, hardware costs money and before diving right in, some users might appreciate being able to try APRS out first. A good, cheap alternative to dedicated 


\section{CHAPTER 3. APPROACHES TO ACCESSING THE APRS NETWORK}

hardware is to use hardware that hams already have. A good choice that will fit the needs is a computer, which most hams are likely to already own. If they don't happen to own a computer, much of this argument is null and void since a computer is required to use both TNCs and to program the specialty hardware. On the computer, amateurs can use software to do the modulation and demodulation, and build or buy a cheap interface to a radio, around $\$ 15$ instead of roughly $\$ 200$ for a piece of dedicated hardware.

This seems to be a route that some are taking and a demodulation scheme that this project explores in detail, but before exploring this in more detail, more information on current systems that operate in this software realm is necessary. Some examples of the software that can be used are George Rossopoylos's Packet Engine [52], Thomas Sailer's Linux Sound Modem [55, 56], and Sivan Toledo's javAX25 [72, 70]. On a computer, even one with minimal resources, there are algorithms that are being used to demodulate the APRS packets. Again, what this project aims to investigate is what improvements can be made to the algorithms of software-based demodulation approaches in order to achieve performance similar to TNCs and dedicated hardware. Improved performance for the software is defined by being able to correctly decode more packets in an efficient, real time, manner. Based on observations in initial analyses where software was unable to decode packets, the hypothesis is made that improvements can be made to software based demodulation.

\subsection{1 javAX25}

Sivan Toledo's javAX25 is very comprehensive software package that handles the encoding, decoding, radio control, and interfacing with sound cards to allow for full use of APRS. However, in addition to just being able to utilize APRS, there is also a test application inside of this package that allows for quick and easy testing of everything in the suite - of the most interest, however, is the ability to test demodulators. Although all of these features were included, the three primarily used in this project were the modulation, demodulation, and demodulator testing. Due to its comprehensiveness and ease of access online through Github, javAX25 was chosen to be the basis for this project [72]. For a complete list of features, the manual can be found in the following reference, and even from the beginning the mission statement the author outlines coincides with that of this research [70]. 
Toledo did some benchmarking of his software and found that running two demodulators in parallel provided the best results. The demodulators were exactly the same; the only difference was that one was processing data after a bandpass filter that was centered around the two frequencies of interest, and the other had a bandpass filter that additionally applied $6 \mathrm{~dB}$ of attenuation at $1200 \mathrm{~Hz}[71]$. Being published in 2012, this is the newest reference in this paper on the subject of AX.25 (aside from the papers published by Finnegan [19, 20]), which provided additional incentive to use this project for this research. As added verification of making the correct decision of what software to use, a very popular Android APRS application written by Georg Lukas uses javAX25 by a direct import [38]. 


\section{Bell 202 Demodulation Techniques}

There are a few primary approaches to demodulating 1200 baud $1200 \mathrm{~Hz} / 2200 \mathrm{~Hz}$ AFSK signals in hardware. However, before talking about the techniques for doing AFSK demodulation, it is worth specifying what type of FSK Bell 202 is. There are two features that are relevant for taking into consideration when demodulating the signal. First is that it is asynchronous, meaning that there is no separate clocking signal as the clocking is embedded within the data signal. Hence the conversation on bit stuffing and clocking energy in the APRS Background chapter. If it were synchronous, there would be two different signal inputs coming into the demodulator; the actual data and the clock. The second characteristic is that the FSK is coherent or continuous. This means that there is a continuous signal at bit boundaries and there are no jumps as the signal changes from one frequency to another as seen in Figure 4.1 as opposed to non-coherent Figure 4.2 which does have these jumps. Another name sometime applied to this method of FSK, coherent FSK, is continuous-phase frequency shift keying or CPFSK [77].

Among the demodulation techniques for coherent FSK are edge detection, correlation, filtering, and phase-locked-loops (PLLs). Each of these will be explained in more detail in the corresponding sections below; however with Correlation and Filtering being very popular and mentioned in many books and applications, there is a lot of overlap so more detailed information can be found in the following references [61, 62, 14, 50, 58, 59]. The following sections in this chapter explain how the demodulation approach works with the software implementation details being saved for the Implementation chapter. However, there will be an additional section at the end of this chapter to discuss some of the potential advantages of using software over hardware. Before talking about all of the techniques a little context as to which onces are being used in the various pieces of hardware can be found in Table 4.1.

Table 4.1: Hardware Demodulation Techniques [31, 18, 16, 33, 43, 2].

\begin{tabular}{|l|l|} 
Device & Demodulation Technique \\
\hline OpenTracker 2 & PLL using XR-2211 \\
\hline OpenTracker 3 & Software time delay correlation \\
\hline Kantronics Kam & Edge detection using TCM3105 Chip \\
\hline MFJ-1278 & PLL using XR-2211 \\
\hline PK-88 & Digital filters using AMD 7910 chip \\
\hline PK-232 & Analog filters \\
\hline
\end{tabular}


CHAPTER 4. BELL 202 DEMODULATION TECHNIQUES

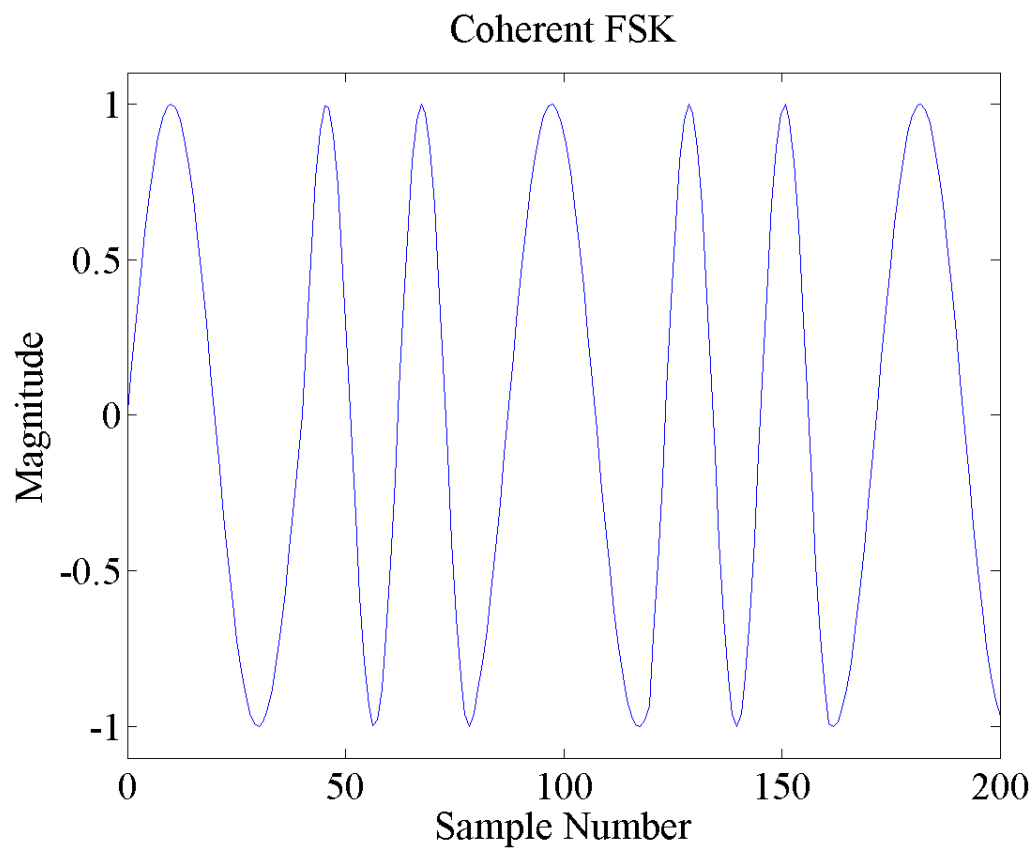

Figure 4.1. Example of a coherent FSK signal.

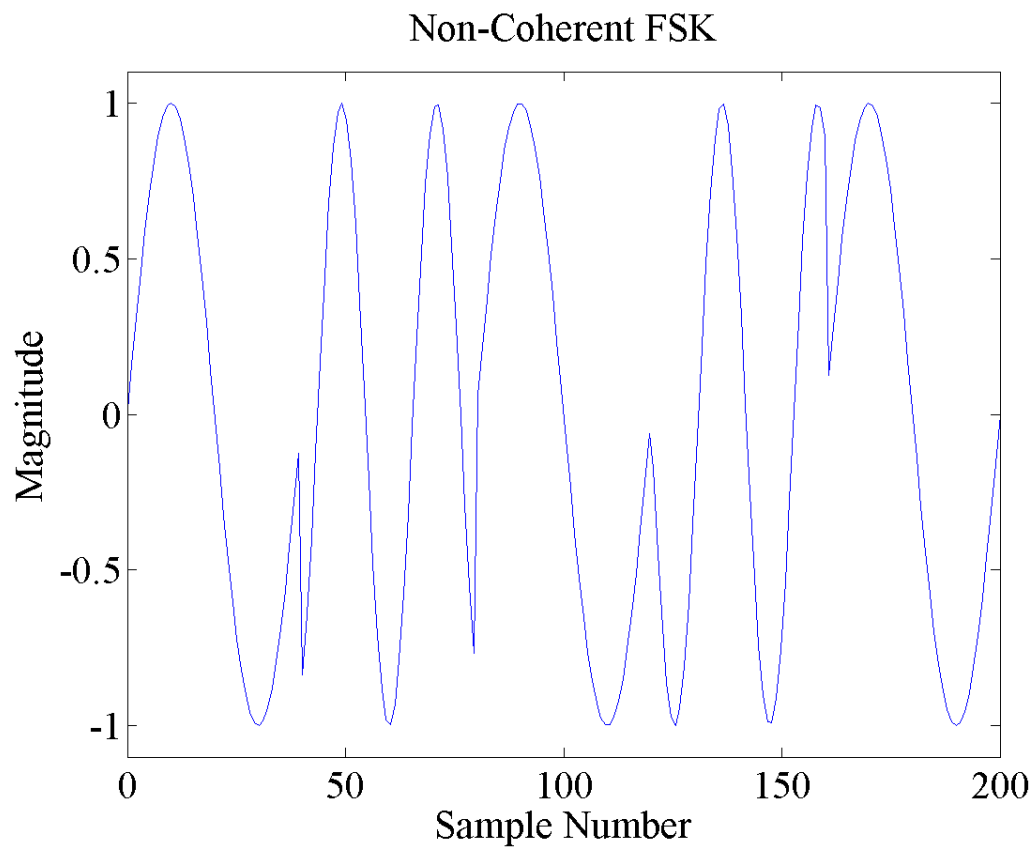

Figure 4.2. Example of a non-coherent FSK signal. 


\section{CHAPTER 4. BELL 202 DEMODULATION TECHNIQUES}

\subsection{Edge Detection}

An edge detection, or zero-crossing, demodulator identifies rising and falling edges in the signal to determine the frequency present. In the TCM3105 chip, which is an FSK modem, rising and falling edges trigger pulses that are at a frequency that is double the input frequency [33]. Although this is how it is done in hardware, it may be easier to understand through the more simple discussion of zero-crossings. The idea is that based off of the time elapsed between zero crossings (rising and falling edges or vice versa) of the signal, one-half the period of the waveform can be measured. Once the period has been measured the frequency can then be easily calculated using the inverse relationship between period and frequency, $f=1 / \mathrm{T}$ where $f$ is the frequency and $\mathrm{T}$ is the period [58]. Just to reiterate, two consecutive zero crossings is only half of the period do to the nature of sinusoidal signals, which is also why the TCM3105 chip outputs pulses at twice the input frequency. As the TCM3105 is an older chip, a replacement is now commonly used in 1200 baud modem projects, and that is the MX614 made by MX-COM [45].

\subsection{Correlation}

A correlation demodulator works through correlating - comparing - an FSK signal with the possible options based off the modulation scheme. In this instance we expect the signal to be either a $1200 \mathrm{~Hz}$ or $2200 \mathrm{~Hz}$ signal and hence the signal will be compared to two internal oscillators, one at each frequency [53]. In practice, the input signal is mixed with each one of the two reference signals and then integrated over the bit period. The results from each of these correlations is then fed into a decision unit. The output of the decision unit is whichever of the frequencies has more power and hence was more prominent in the signal. The basic block diagram can be seen in Figure 4.3. Correlation is the current method used in Toledo's javAX25. An alternative implementation of a correlator is to have a delay line instead of an internal oscillator. This delay line can delay the input by the time for one period of an expected frequency (i.e. $1 / 2200 \mathrm{~Hz}$ ) to elapse and then this delayed signal can be multiplied by the original signal [58]. Essentially, the delayed signal becomes the internal oscillator in this example (see Figure 4.4). Side note, this would work better for higher frequencies than those here since the delay line would be about 145 miles long if using 


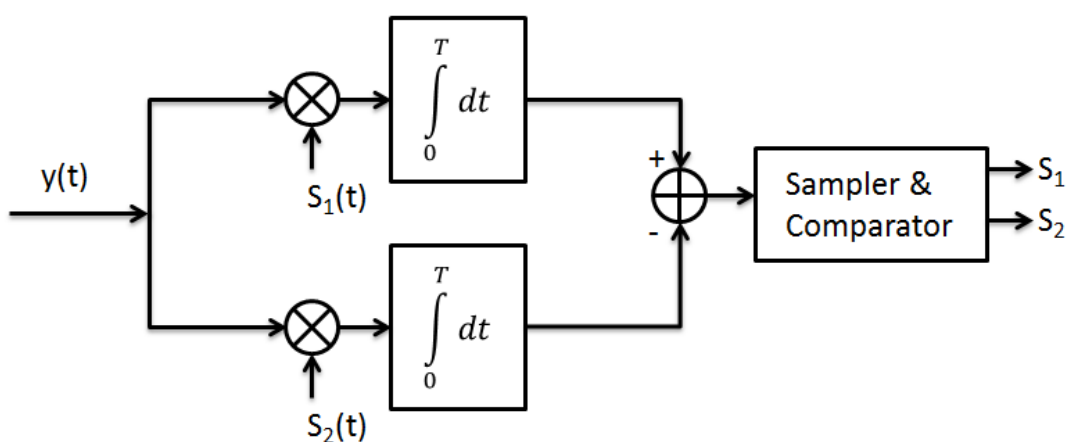

(a) Correlation receiver

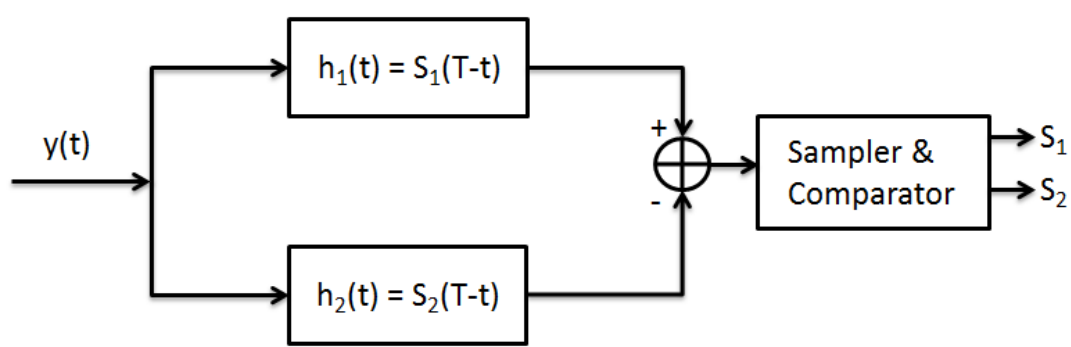

(b) Matched filter receiver

Figure 4.3. Block diagrams for (a) A Correlation Receiver and (b) A Matched Filter Receiver [14].

copper wire for the $1200 \mathrm{~Hz}$ tone [40]. However, this is possible in software since buffers can be used.

\subsection{Filtering}

Much like the correlation demodulation approach, a filter based demodulator operates on knowing the expected frequencies in the FSK signal. For our case of $1200 \mathrm{~Hz}$ and $2200 \mathrm{~Hz}$ frequencies, a filter will be set and centered about each one of these frequencies. The input signal is passed to each one of these narrow band pass filters and then the power of each of the signals out of the filters is fed to a comparator. The stronger of the two frequencies is the one that must have been present in the original signal [74]. One example of a filter that can be used is a Finite Impulse Response (FIR) Filter. The block diagram for this approach can 
CHAPTER 4. BELL 202 DEMODULATION TECHNIQUES

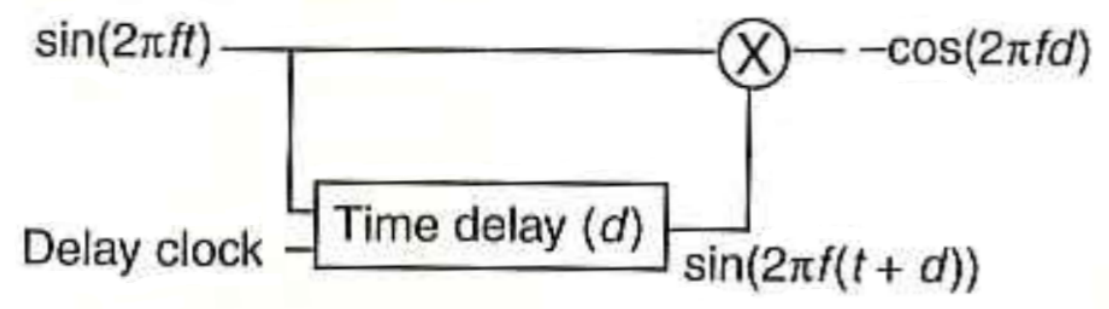

Figure 4.4. Block Diagram of a time delay correlator [58].

be seen in Figure 4.3, and the general structure is very similar to correlation. This method seems to be prevalent in both hardware and software based approaches. For instance, if one examines the schematic for the PK-232 MBX, two parallel filters can be seen [31]. Rossopoulos's Packet Engine measures the energy on the two modem frequencies using filters to do the demodulation. Sailer's Sound Modem also uses a matched filter demodulation [54] whose algorithm was then reused on a micro controller by Holder [29]. Additionally, AMD produced an FSK Modem chip that used digital filtering for demodulation [16]. All of these independent uses make this look like the most prominent approach for demodulation.

\subsubsection{Discrete Fourier Transform}

One example of a digital filter is a Discrete Fourier Transform (DFT). A discrete Fourier Transform is one implementation of Fourier Transform that is executed on discrete samples similar to what is present in a digital audio file. Once a Fourier transform has been applied on a signal the output is a relative power versus frequency. With this data, whichever frequency (either $1200 \mathrm{~Hz}$ or $2200 \mathrm{~Hz}$ ) is more prominent is the symbol which must be present in the bit period, and hence can be used for the demodulation; however, Fourier Transforms are computationally intensive.

\subsubsection{Goertzel Algorithm}

Computing a discrete Fourier transform is more reasonable computationally than a full Fourier transform, which is a continuous integral as opposed to having discrete terms [80]. However, even the results from the DFT have more data than is needed to do the demodula- 
CHAPTER 4. BELL 202 DEMODULATION TECHNIQUES

tion since the results will be a spectrum of powers over a range of frequencies [78, 79]. A more simplified and specific approach can be used. The Goertzel Algorithm evaluates the coefficients and corresponding powers of the individual frequencies of $1200 \mathrm{~Hz}$ and $2200 \mathrm{~Hz}[81,17]$. This approach, sometimes called a Goertzel Filter, means that no additional computation is wasted on computing frequency power data that is not relevant, making it faster and a vast simplification to the DFT [57].

\subsection{Phase Locked Loop}

Another option for determining the frequencies present in the original data carrying signal, and hence the actual data in the signal, is to use a Phase-Locked Loop (PLL) [3, 48, 39]. There are a few different approaches for utilizing a phase locked loop. The basic idea is that there is an internal oscillator and the input (the received signal) is used to influence this oscillator [21] . The input signal and the reference oscillator signal are integrated and this output is used for feedback to the internal oscillator (hence the loop portion of PLL) [51]. The convenient thing about monitoring the phase of the signal so closely and being able to stay locked onto it is that the frequency must also be known and this is the portion that is really of interest. A block diagram of a phase locked loop can be seen in Figure 4.5. There is a chip produced by Exar that does FSK demodulation and tone detection using a PLL, for which the model number is XR-2211A [18]. A circuit diagram of using the XR-2211A for Bell 202 can be seen in Figure 4.6 which also shows some of the primary items needed for a PLL including the phase detection (integrator) and the VCO (Voltage Controlled Oscillator).

\subsection{Additional Benefits of Software Based Decoding}

Software is flexible. As Bergquist mentioned, it was only a matter of time before hams developed a bond between computers and radios using TNCs and it is time to take it a step further and leverage the capabilities of a computer even more [5]. Any one of the aforementioned algorithms can be used on the same hardware without having to add additional discrete components, new Integrated Circuits (ICs), or make modifications to a Printed Circuit Board (PCB). There are two benefits of using software instead of dedicated hard- 


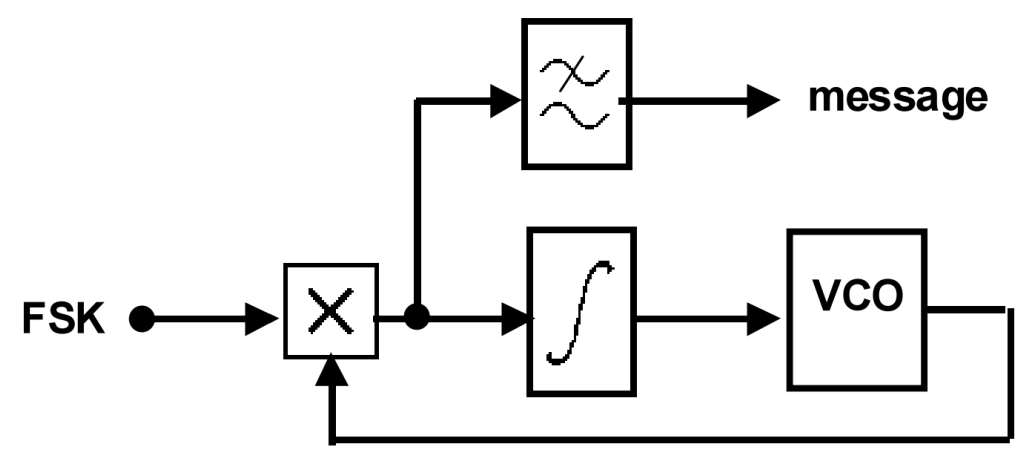

Figure 4.5. Block diagram of a PLL demodulator [51].

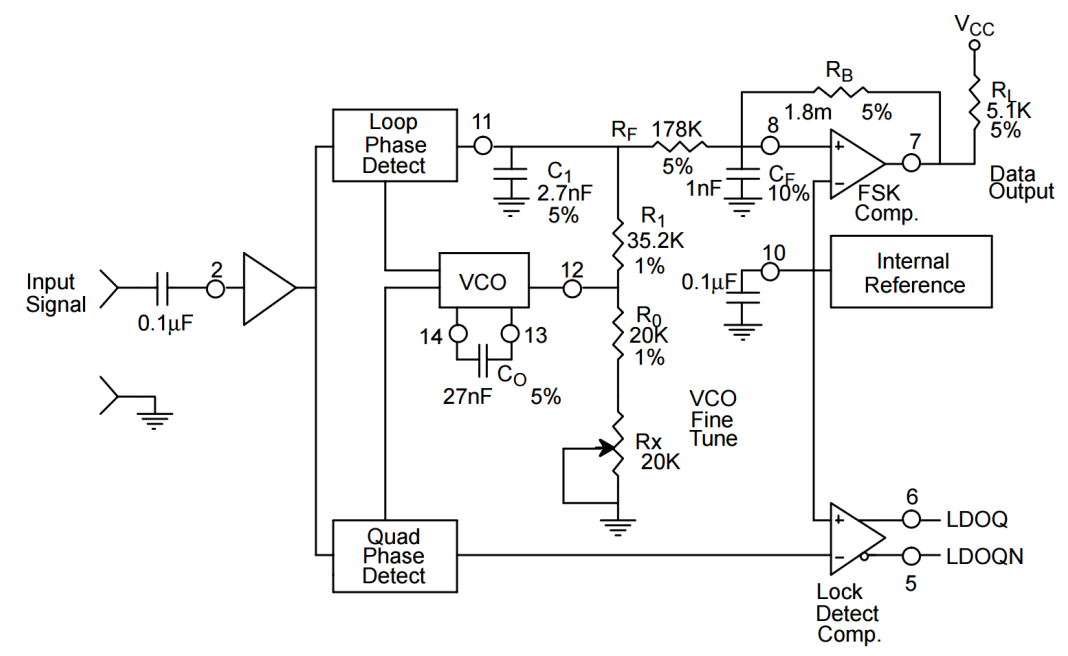

Figure 4.6. Circuit connection for FSK Decoding of Bell 202 Format using an Exar XR$2211 \mathrm{~A}[18]$. 
CHAPTER 4. BELL 202 DEMODULATION TECHNIQUES

ware that this research will investigate; first exhaustive search of a signal through the use of buffers, and second, the ability to be able to run multiple of these demodulation approaches in parallel.

\subsubsection{Exhaustive Search of Incoming Signal}

Using software the input signal can be buffered in the program and then searched for a signal. Since there is not a separate data and clock signal, there could be a case when the clocking is improperly selected. Using an approach of buffering data that may contain a valid packet, the software can step through the data trying every possible clocking option.

\subsubsection{Taking Advantage of Parallel Demodulation}

Another advantage of using software for decoding these AFSK signals is being able to apply multiple demodulation techniques. Once the data is collected and converted to a digital form there is no reason, other than computation limitations of the host computer, not to run multiple algorithms in parallel in order to be able to demodulate the maximum number of packets possible. Although there are packets that every algorithm is able to decode, there are also packets that some approaches can decode while others can not. Through using multiple demodulators in parallel and de-duplicating the results, even more packets can be correctly decoded. 


\section{Demodulation Challenges}

While analyzing different demodulation algorithms and trying to improve their performance, there were a few phenomena that were observed that made correct demodulation more challenging. The following items created difficulty and can all be attributed to the fact that APRS uses RF and hence is susceptible to all the items relating to an RF transmission.

The main challenge in decoding the APRS data was the fact that the digital stream is converted to an audio signal and then transmitted over RF. The addition of RF adds a whole plethora of obstacles which can include Path Loss, Multi-path, Fading, Doppler effects, Co-channel interference, Interference and Noise, and Foliage [24]. A few items which

are important to note, due to the fact that some of the algorithms had to be coded to tolerate them are: DC Offset, Noise, and Emphasis.

\subsection{Challenge: DC Offset}

An audio signal can be characterized by a sine wave. In order to get different sounds the frequency of the sine wave is changed and in the context here, the two frequencies are $1200 \mathrm{~Hz}$ and $2200 \mathrm{~Hz}$. Since an audio signal is a sine wave, the average value should be zero. The zero value is commonly referred to as the ground of the audio signal, and as the definition would imply the signal should spend the same amount of time above ground as it does below ground. As the performance of zero crossing algorithms were investigated it was evident that this was a challenge. If one assumes that the signal is centered about zero, and it is not, the logical decisions made with this incorrect assumption will hence not hold true. Figure 5.1 shows that the signal is not centered around zero. This lack of the signal being centered around 0 (or ground) is why it is said to have a DC offset. It can be noticed in this figure that near the center there are time periods that would be both much longer and much shorter than those expected from subsequent zero crossings during demodulation due to this DC offset effect. 


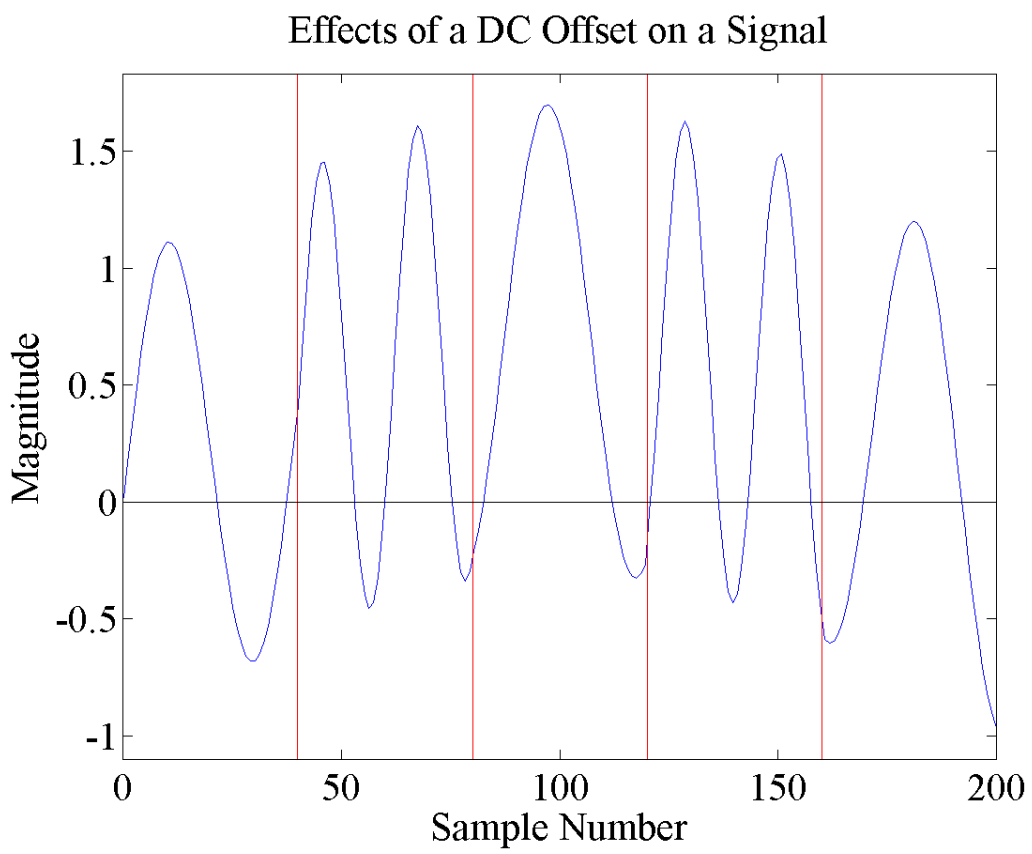

Figure 5.1. An example Bell 202 signal with DC offset problems.

\subsection{Challenge: Noise}

First, a definition of noise in the context of this research: noise refers to unwanted electrical signals present in electrical systems [62]. Since the data is an AFSK signal that is then frequency modulated, there are two distinct steps where noise can be introduced, but must be kept to a minimum to increase the chances of the data being properly transferred. Hence, a large signal to noise ratio (SNR) is preferred. This is not the case for only APRS but with all wireless technologies. One cause of having an increased effect from noise and hence a lower SNR is increased distance between the transmitter and receiver. An example that many are probably familiar with is as a client gets farther away from a wireless access point the bandwidth decreases. This happens because the signal strength drops off at $1 /$ distance cubed [65]. What was noticed when some of the algorithms were being debugged was the random noise embedded in the signal caused significant problems.

One such example of this is if the noise happened to also take on the form of a sine wave and the algorithm locked onto that frequency instead of the $1200 \mathrm{~Hz}$ or $2200 \mathrm{~Hz}$ signal that was wanted to be decoded. Alternatively, if the noise was just random and jostled the signal in 


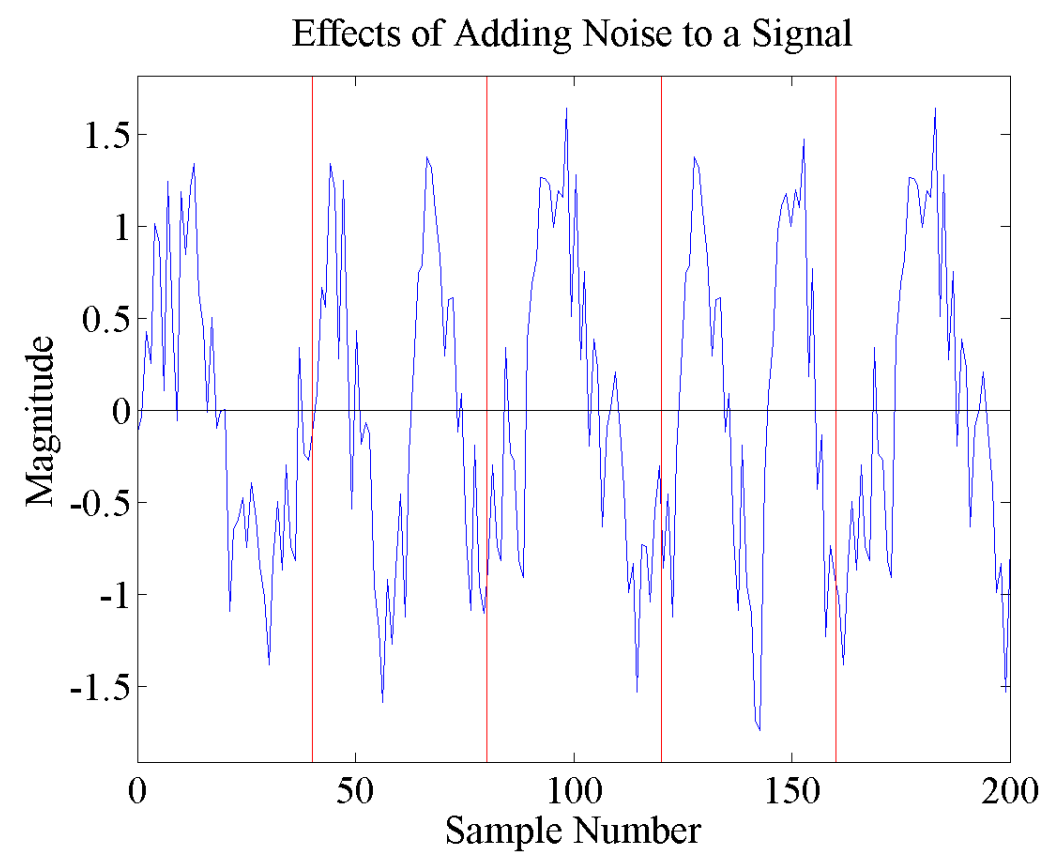

Figure 5.2. An example Bell 202 signal with white noise added. The additional zero crossings at sample 9 in the first bit period and sample 169 in the last bit period will make these $1200 \mathrm{~Hz}$ bit periods look more like $2200 \mathrm{~Hz}$ signals.

the correct spot $1200 \mathrm{~Hz}$ tones might look like $2200 \mathrm{~Hz}$ (this ended up being fairly common) or vice versa. An example of what noise may look like on the original signal can be seen in Figure 5.2.

\subsection{Challenge: Emphasis}

Due to the fact that this is AFSK data being transmitted over a voice channel, emphasis becomes a concern. Preemphasis is when the the higher audio tones in a Frequency Modulated (FM) signal are intentionally increased and deemphasis is when that process is reversed on the receiving end to return the audio to a flat level. Why emphasize the audio signal? This process of emphasis is not necessary, but the effects are desirable since it increases the signal to noise ratio in the RF signal by having the higher audio tones preemphasized [23]. The reason this is a concern is because the adoption of emphasizing and deemphasizing APRS signals is inconsistent. Some radios have data ports which bypass the emphasis step 
CHAPTER 5. DEMODULATION CHALLENGES

\section{Deemphasized Signal that was not Preemphasized}

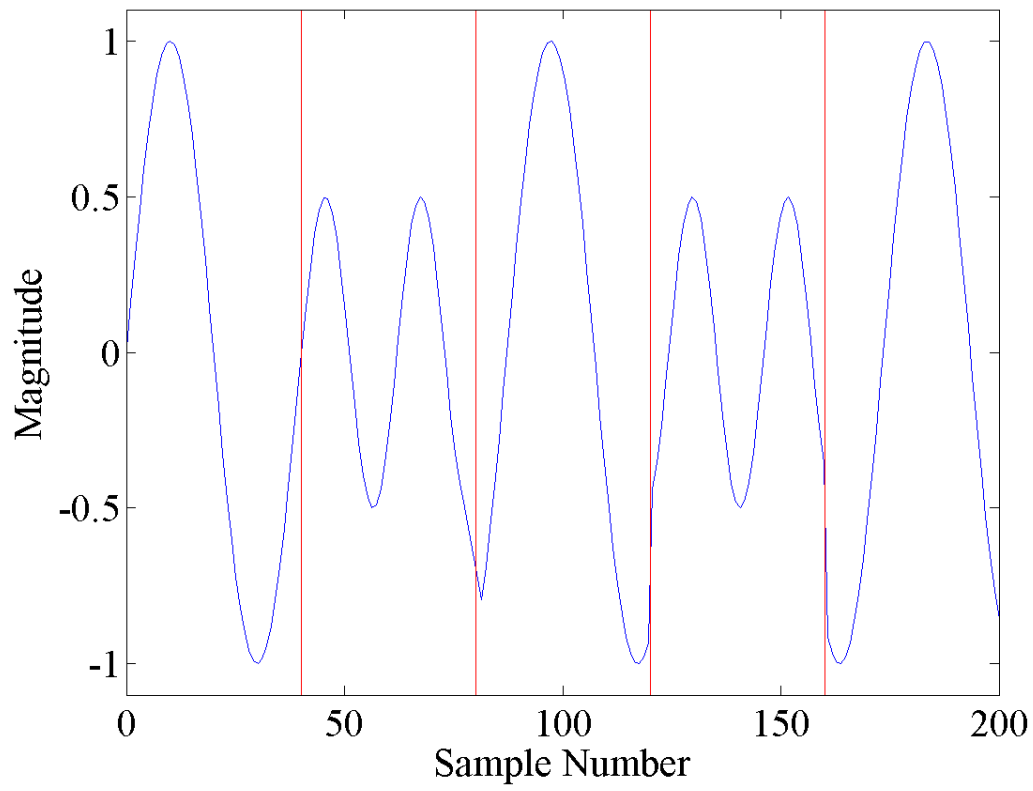

Figure 5.3. An example signal that was not preemphasized, but was deemphasized.

while other APRS users utilize the microphone and speaker ports of a radio which would utilize emphasis. This would mean that a signal transmitted out of a radio with a data port may not be emphasized, but then when received through the speaker out of another radio would be deemphasized. The effects of a non-emphasized signal being deemphasized can be seen in Figure 5.3. This causes problems with the demodulation because it can not be assumed that the relative powers of each frequency will be equal since they will have different magnitudes in this case. 


\section{Demodulator Benchmarking}

In order to compare the results of the different demodulators a method of benchmarking must be instituted. Each one of the demodulators was tested using multiple audio files that have different characteristics. These different files as well as the advantage of using them in the benchmarking are explained in their corresponding section. The winner of each of the benchmark files is determined by which demodulator could demodulate the most packets out of the file. It is worth noting that for each test audio file a sample rate of $48000 \mathrm{~Hz}$ was standardized on since it works out nicely to 40 samples per bit period for 1200 baud digital communications which made conceptualizing the data easier.

\subsection{Plain, Straight, and Clean Packets}

These audio files were just what the title implies - straight packets. What is meant by straight packets is that they are pure "perfect" $1200 \mathrm{~Hz}$ and $2200 \mathrm{~Hz}$ tone audio samples. There is no noise introduced through artificial or natural means such as that introduced through the intrinsics of using RF. Although these files do not provide meaningful results for hardware or already implemented software solutions (since these devices should be able to decode every packet in the audio file) it still provides a good starting point for getting new algorithms up and running. Two of these clean files were generated. One was generated from an OpenTracker creating packets with a counter and the text "The quick brown fox jumps over the lazy dog". This audio file contained a total of 40 packets each separated by $30 \mathrm{~ms}$ and proved to be short enough to allow for quick cross checking as modifications were made. The second file was generated in software using Toledo's javAX25 package. All that is relevant at this point is that it has perfect levels $(1200 \mathrm{~Hz}$ and $2200 \mathrm{~Hz}$ tones are at the exact same level) and contains 200 packets each separated by $300 \mathrm{~ms}$ (this was chosen so that the space between packets was sufficient to be heard) making the file quite a bit longer. A sample from the 200 packet audio file can be seen in Figure 6.1. 


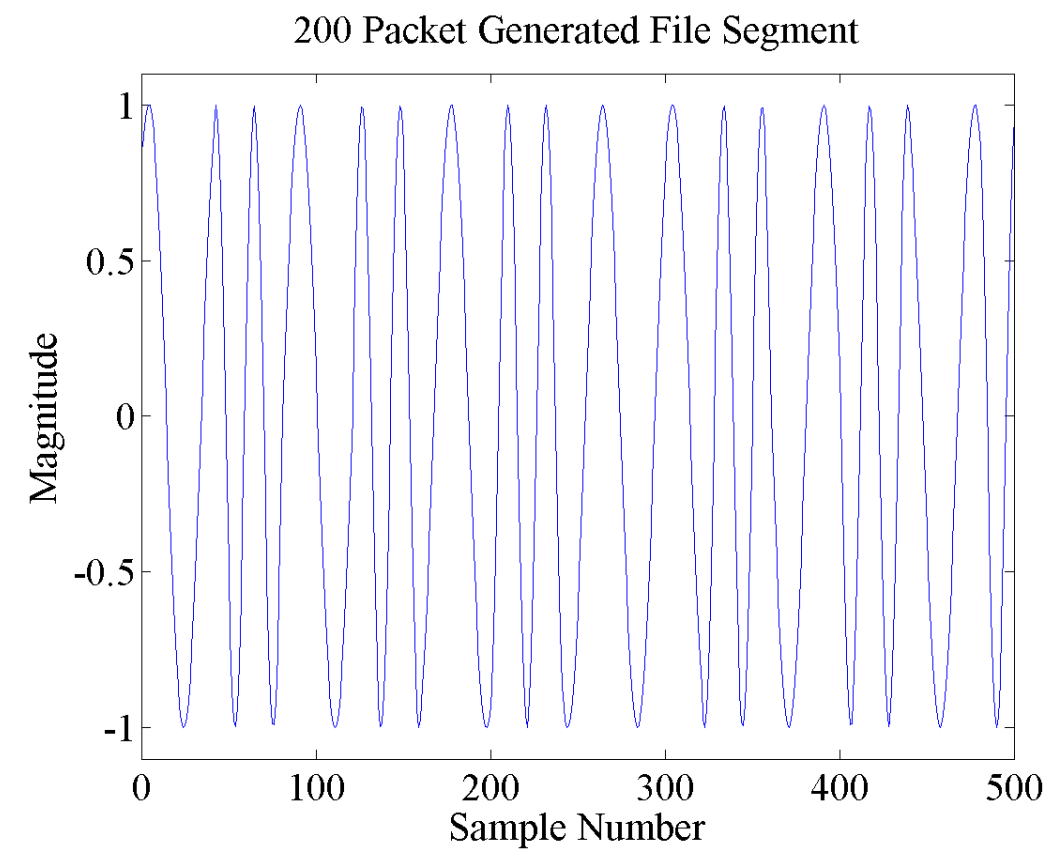

Figure 6.1. Example of the AFSK signal present in the 200 packet generated file.

\subsection{White Noise Testing}

The second test file that was used on the demodulators was the 40 packet OpenTracker file mentioned in the previous section with added artificial white noise. An advantage of this file is that its contents are known, it contains 40 packets, while still being a reasonable benchmarking file because the later packets in the file are buried in noise. No demodulator could demodulate all the packets out of the file as the noise was increased all the way up to a signal to noise ratio (SNR) of 0.5 , making the magnitude of the white noise twice that of the original signal. There were total of 10 steps of increasing noise meaning that at each noise level there were approximately 4 packets. Using this information on how the test file was created, the total number of packets decoded from this file in addition to the calculated SNR will be presented in the results section. This noise was added to the original audio file using the audio editing program Audacity [42]. Although this file does not directly characterize what is introduced by $\mathrm{RF}$, it provides a reasonable test simulating the effects of a decreased SNR on the audio signal. An example segment from this white noise added file can be seen in Figure 6.2. 
CHAPTER 6. DEMODULATOR BENCHMARKING

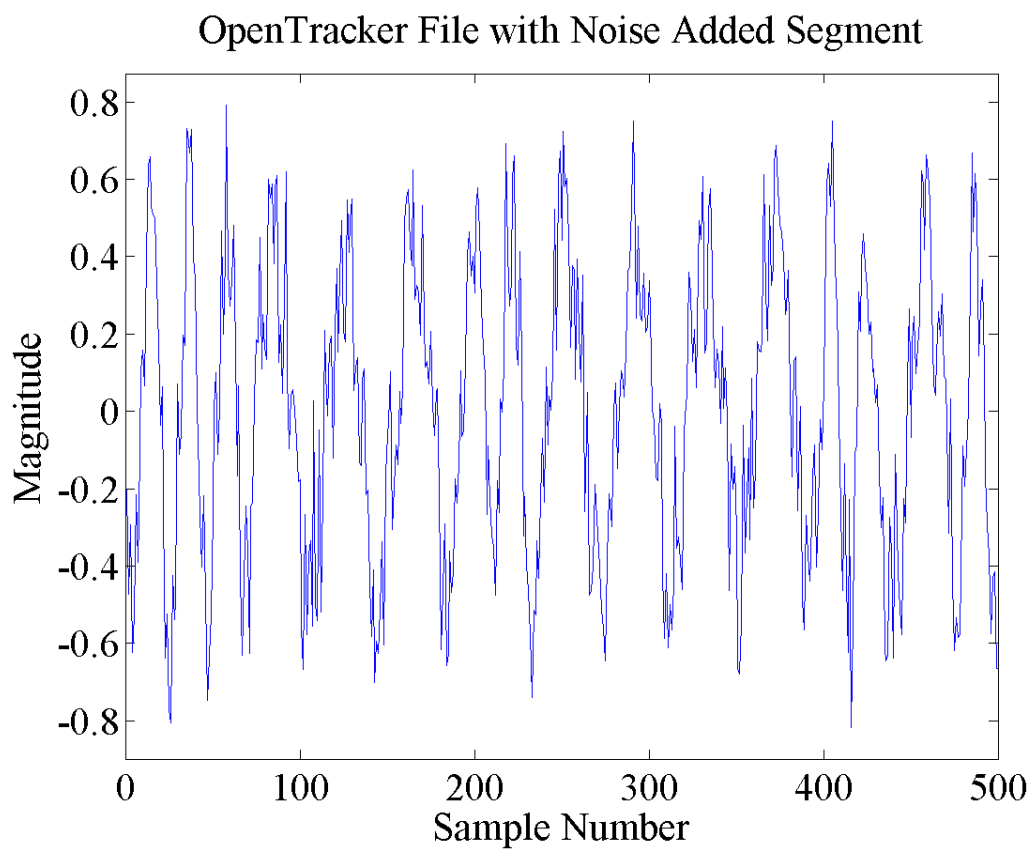

Figure 6.2. Example of a generated AFSK signal with artificial white noise added.

\subsection{Los Angeles APRS Test Recordings}

This next benchmark is the de-facto benchmark for APRS modems. The idea behind it is simple, yet it provides a very comprehensive test. The author of this file recorded on air APRS traffic in the Los Angeles Area for 45 minutes. He then removed segments of no traffic and condensed 45 minutes of live recording down to about 25 minutes [63]. This recording is relevant because it is real traffic which contains all of the real life situations including stations close to the receiver, far from the receiver, moving, stationary, different transmit power levels, different hardware, and varying content just to name a few. One disadvantage is that since it is just a random recording of traffic on the air, there is no definitive answer of how many packets are in the file. By listening to the first 3 minutes and extrapolating those results to the length of the file it is estimated that there are 1463 packets total. This is considered the file use for benchmarking in the community and when people discuss the performance of their demodulator, they quote it in how many packets they were able to successfully decode out of this file. An example segment from this off air recording of APRS traffic can be seen in Figure 6.3. This is just one of the audio files on 
CHAPTER 6. DEMODULATOR BENCHMARKING

Los Angeles Recording Segment

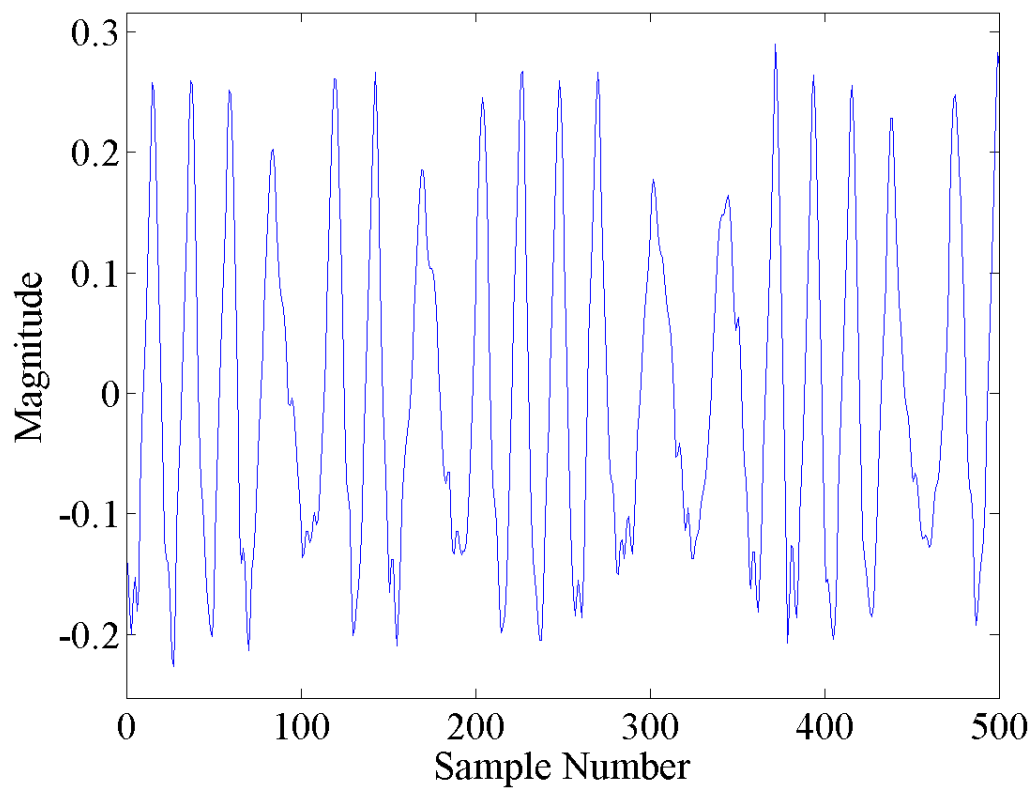

Figure 6.3. Example of the AFSK signal in Track 1 of the Los Angeles Recording Test File.

this author's test CD and is in fact the first one on the CD, so it will be referred to as Track 1. This was the the file that was most important to the testing, but the author also has a second version of this file in Track 2. The only difference between Track 1 and Track 2 is that an audio filter was used to create Track 2 which had the result of being a deemphasized version of Track 1. A quick note about Track 2 is that the processing performed to create it had other undesirable side effects including the white noise between packets being at a much higher magnitude than the packets themselves. 


\section{Testing}

In order to be able to evaluate the results from this research, each demodulation technique considered needs to be tested and the number of packets that each technique was able to successfully decode needs to be compared. From this analysis, it will be seen which techniques are effective and are able to decode relatively more packets as opposed to those that decode fewer. In order to validate the software techniques, results for dedicated hardware will also be collected for comparison. The testing for both the dedicated hardware and the software algorithms will be described in the corresponding sections below.

\subsection{Hardware Testing Setup}

The testing setup for the hardware is fairly simple since they are basically black boxes that just need to be supplied with the correct inputs. Each piece of hardware has two connections; one is the radio port, and the other is the serial connection. As the name implies, the radio port is used to be able to interface with the radio. This port has connections such as transmit audio, receive audio, push-to-talk (PTT), supply voltage (VCC), and ground. A digram of the common radio port can be seen in Figure 7.1 and found in any manual including those of Argent Data [68]. Since this was common between multiple pieces of hardware, a simple break-out board was created that allowed for a more universal audio transport mechanism of $23.5 \mathrm{~mm}$ tip-ring-sleeve connectors (one audio in and one out), and also a $2.5 \mathrm{~mm}$ barrel jack for power (Figure 7.2). This was much simpler than actually interfacing with a radio since the audio could just be played from a laptop into the device using a standard $3.5 \mathrm{~mm}$ tip-ring-sleeve cable (Figure 7.3).

\subsubsection{Setting TNC Audio Levels}

During the testing, setting the audio level to be precisely correct for optimal performance would have been ideal. However, there is language in the user manuals for the various TNCs that is definitely up for interpretation. Such as in the MFJ-1278 manual which reads: "Continue to advance the volume control until there is approximately twice as much audio present at the receiver output 〈as the minimum〉" [43]. Additionally the manual for the Kantronics KamPlus says: "Increase the volume control slightly from this point" [35]. 
CHAPTER 7. TESTING

\section{Kantronics VHF DB-9 Connector}

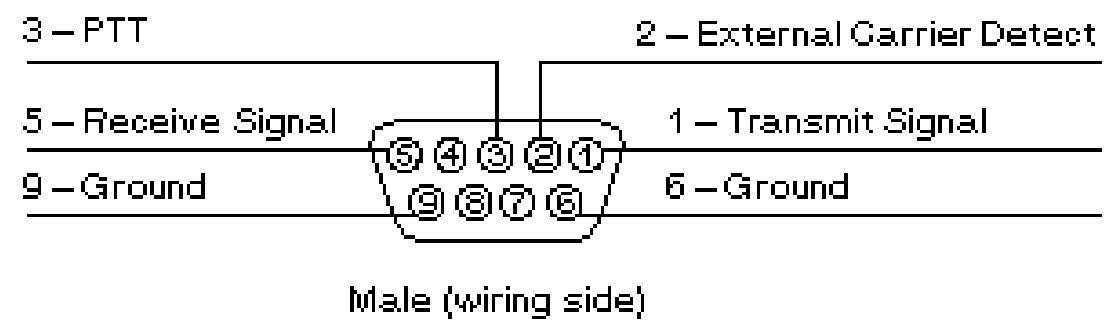

Figure 7.1. Example Radio Port pin out for Kantronics, also consistent with others including OpenTrackers [41].

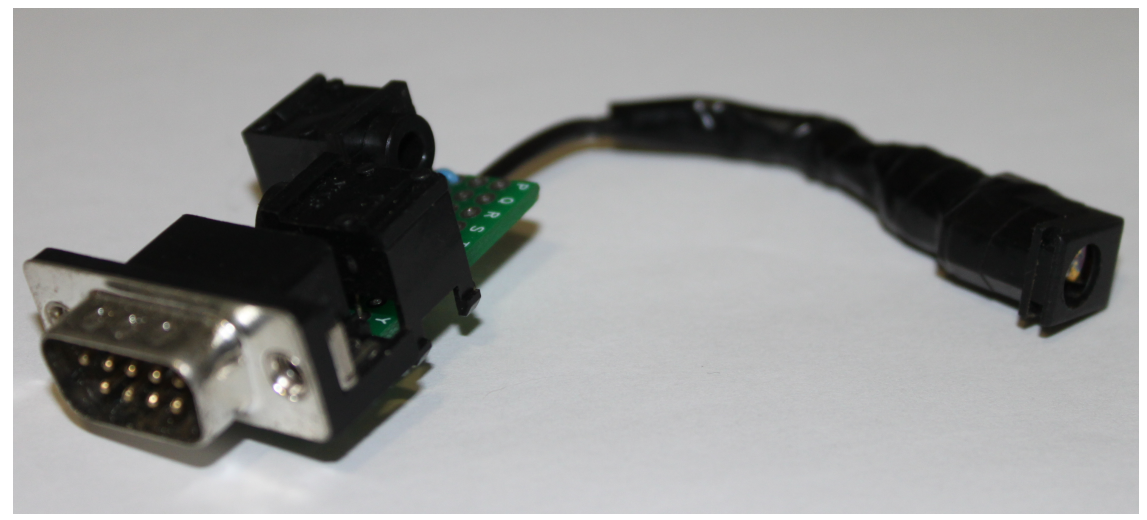

Figure 7.2. Break-out board fabricated for hardware testing.

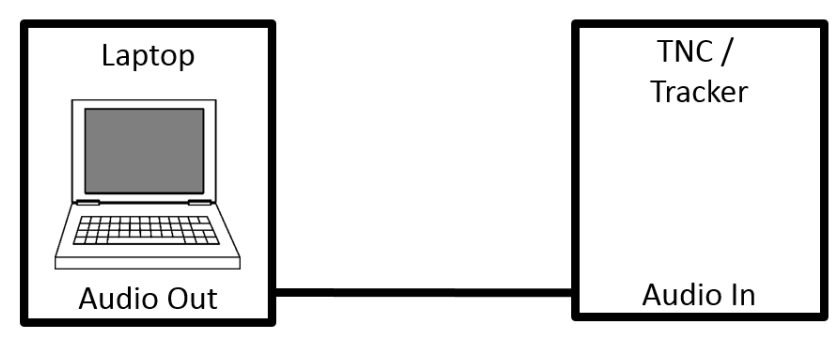

Figure 7.3. Block diagram of the test setup used for testing APRS hardware. 
CHAPTER 7. TESTING

Table 7.1: Minimum Optimal TNC Input Audio Levels [35, 43, 2, 31].

\begin{tabular}{|l|c|c|c|c|}
\hline TNC & KamPlus & MFJ-1278 & PK-88 & PK-232 \\
\hline Level (Vpp) & 0.5 & 0.25 & 0.15 & 0.35 \\
\hline
\end{tabular}

The volume level that was used for the audio provided to the TNCs was setting the laptop system volume to $50 \%$ which worked out to about $1.1 \mathrm{~V}$ peak-to-peak (Vpp). After doing some deeper reading of the individual TNC user manuals and verifying the expected results that were outlined, the approximate minimum optimal audio levels can be seen in Table 7.1 .

\subsection{Software Testing Setup}

Included within the javAX25 suite was a testing application that could both generate and decode packets. However, it was limited to only being able to specify one audio file and one demodulation algorithm. Using this test file as a basis, a new testing application was created that allowed for the multiple demodulators to be compared side by side against multiple audio files with a single run of the application. In addition to the output being printed to the console, it was saved to a file. Having these features in the testing application allowed for a much more streamlined analysis of all the algorithms collectively and tuning individual algorithms. One very convenient aspect of programmatically testing is that it is very easy to add a loop to try a range of tuning parameters and then look at the results to decide what is the best option. All of the results listed in the following chapter are from the testing application and mechanism described here. 


\section{Implementation}

This chapter will go through all of the implementation details of each demodulator implemented. They will be presented in order of complexity, with the more intricate ones presented last. This also will introduce them mostly chronologically since naive approaches allowed for more insight to be gained into the javAX25 software package, as well as APRS packet demodulation, before implementing more complicated algorithms. In addition to giving a brief overview of each implementation some performance data will be provided, but all of the data will be presented in the Results chapter. As mentioned in the Demodulation Techniques chapter, javAX25 was selected because of its availability and as such all of these implementations can be found in this author's Fork of the javAX25 GitHub repository in the following reference [10].

\subsection{Strict Zero Crossing Demodulator}

This approach used the technique of finding zero crossings and then using those to determine the period; and frequency. For $1200 \mathrm{~Hz}$ and $2200 \mathrm{~Hz}$ tones, zero crossings are expected every 833us and 455 us respectively. If the resulting frequency after calculations was above $1700 \mathrm{~Hz}$ it was assumed that a mark was present in the signal and if lower than $1700 \mathrm{~Hz}$ a space must be present. Each zero crossing was found by determining if the signal was negative and changed to positive or if it was positive and changed to negative. Although this algorithm was only able to decode a little over half of the packets as some of the other algorithms, it proved to be an important stepping stone into javAX25 and allowed for preparation into restructuring the project for added modularity of the filtering.

\subsection{Floating Ground Zero Crossing Demodulator}

Building on the strict zero crossing algorithm the next zero crossing demodulator tried to use some more intelligence in finding the zero crossings through additional processing. One reason that the strict zero crossing approach was thought to have relatively poor results was due to the previously introduced challenge of DC offset. If the signal does not actually cross zero then it will be very hard to find the zero crossings. This new zero crossing method 


\section{CHAPTER 8. IMPLEMENTATION}

keeps a window of history (it was arbitrarily chosen to be one bit period) and from this collection of samples the average is taken to use this as the ground - or zero value. Instead of checking to see if the signal crosses zero, the signal is analyzed for going from either above to below or below to above this average value. This ended up having worse results than the strict zero crossing demodulator. This was due in part to the fact that $2200 \mathrm{~Hz}$ signals - even when properly centered around zero - will not have an average of zero since it can not complete two full periods within one bit period, which tainted the average. Referring back to Figure 2.1 it can be seen that the second bit (samples 40-80) would have a positive average, not zero, since at the end of the bit period (sample 80 ), this $2200 \mathrm{~Hz}$ tone still has 60 degrees to go to finish its current cycle.

\subsection{Windowed Zero Crossing Counting Demodulator}

With a good handle on utilizing zero crossing, a new approach was taken to keeping history. Instead of using the history to calculate where "zero" is, a new question was asked, "What if how many zero crossings within one period is observed?" If a window slightly shorter than one bit period is selected, then if there are only two crossings within the window that will correspond to a $1200 \mathrm{~Hz}$ symbol being present. More crossings than two means that a $2200 \mathrm{~Hz}$ symbol must be present. The thought behind taking this approach is that it would give some additional resiliency to noise by finding the average during that bit period through utilizing multiple zero crossings instead of individually analyzing every zero crossing.

\subsection{Peak Detection Demodulator}

After making a simple zero crossing overly complicated, it was decided that maybe a different approach should be taken, specifically to look at a different part of the signal. It was considered that perhaps better performance could be achieved by looking at the peaks in the signal instead of the noisy zero crossing around ground, or not around ground if there are DC offset problems. Conveniently, the difference between two consecutive peaks will be equal to the period of the underlying signal. Although the methodology is the same as the zero crossing for converting the period to the actual frequency, it was perceived that this would give better results. It turns out that this method did not work as well as hoped due 


\section{CHAPTER 8. IMPLEMENTATION}

to the fact that local peaks were commonly discovered from the noise instead of the actual peak in the transmitted signal. More analysis into selecting a proper filter may give this approach better results.

\subsection{Derivative Zero Crossing Demodulator}

After a failure with the peak detection demodulator, a new approach was taken to finding "peaks." Instead of actually looking for the peaks, the zero crossing demodulator was revisited with a new spin. Instead of using the raw samples for determining the frequency using zero crossings, the derivative was to be used. The derivative was calculated by doing the same averaging as in the strict zero crossing approach and then subtracting the current average from the average two samples prior. It was thought that this would solve the DC offset problem for sure, but it turns out that this was not the larger problem. The problem was with using the zero crossing approach and this derivative implementation ended up having very similar results to the strict zero crossing with which of these two was better changing based off of pre-filtering. This can be attributed to the change in emphasis caused by taking the derivative.

\subsection{Goertzel Filter Demodulator}

Finally moving away from approaches utilizing zero crossing methodologies, an approach using Goertzel filters was implemented. The implementation was very simple and corresponds with that outlined in the Demodulation Techniques chapter. Since it has to be applied onto a set of data, a window size that was equal to one bit period was originally selected so as to make sure that the data being processed was only that of one frequency, but after analyzing the effect of the window size on performance, a window size of slightly longer than a bit period ended up being better. The optimal size was tested to be 135 percent of a bit period, and the reason why this worked better is because it gave more signal in the window for the filter to lock to. Essentially, the window was only extended 18 percent on each side of a bit period. This over-extension of the window is what led to being able to exceed the performance of the original correlator on unfiltered data. 
CHAPTER 8. IMPLEMENTATION

\subsection{Phase Locked Loop Demodulator}

Next, the PLL demodulator was implemented. Using Lutus's python based software PLL initial testing was performed to see how it would work for tracking AX.25 signals [39]. Once the parameters were tuned sufficiently that it seemed to be staying locked onto the signal, it was ported over to java and actually run as a demodulator. Once inside of the javAX25 framework additional tuning was done programmatically instead of manually to further fine tune the performance. The final results were that it was not the winner, but comparable to the other top contenders.

\subsection{Mixed Preclocking Demodulator}

Finally, with numerous simple algorithms implemented, it was time to try something much more complicated and also only possible in software. This approach and the name preclocking comes from an abbreviation for predetermined clocking where packets are analyzed a whole packet at a time. The start and end are found and then the clocking, and hence bit boundaries, are predetermined before the actual demodulation takes place on a baud by baud basis as opposed to a sample by sample basis. Each one of the preceding algorithms was on a sample by sample basis, meaning they had to make their best determination of bits elapsed using a little bit of history.

There are five steps to the demodulation in the Mixed Preclocking Demodulator. It was speculated that processing one packet at a time with the correct clocking to demodulate bit by bit would allow for very accurate demodulation.

1. Flags are found in the signal so that the demodulation can happen one packet at a time instead of just blindly trudging forward through the packet sample by sample.

2. The derivative of the whole packet is taken to determine the zero crossings.

3. Frequency transitions are extrapolated from the derivative data.

4. The frequency transitions found in the packet are used to determine the clocking (bit boundaries). 


\section{CHAPTER 8. IMPLEMENTATION}

5. The tone demodulation is done on a baud by baud basis.

Although it was hoped that the results would be phenomenal, there were so many different methodologies being used that this preclocking demodulator was very difficult to tune. For instance, the flags were found using the correlation approach, the transitions using a derivative, and the final demodulation using the zero crossings. What were thought to be advantages ended up being the challenges, but as predicted it did well enough to still be considered one of the successful implementations. The intricate nature of this demodulator made it delicate, which was noticed during the testing through the fact that it would not decode any packets unless a bandpass filter was used on the incoming data.

\subsection{Goertzel Preclocking Demodulator}

After the first attempt at a preclocking approach, it was thought that perhaps using only one methodology to perform all the different steps of demodulation would be at the very least simpler, and hopefully better. The perception that it might be better came from the fact that there was only one item to tune, the Goertzel Filter which had already shown

good performance. Instead of having to worry about noise affecting zero crossings and the derivative potentially adding emphasis problems, only the filter had to be considered. Unfortunately, the number of packets that this method decoded was not as many as the first Goertzel approach or the previous preclocking. This was due to the fact that even though there was one underlying algorithm it was used in three separate instances, and each needed slightly different tuning. The three instances were for flag detection, frequency transition detection, and the the final bit by bit demodulation.

\subsection{Goertzel Exhaustive Preclocking Demodulator}

The final algorithm implemented was just a matter of verification, and another one that could be performed only in software. Instead of analyzing packets one at a time using flags as the start and end points, a whole array of data that had a length equal to the number of samples that a packet of the maximum length would have. Every time a few more samples came in, every single clocking was attempted on the large array of data just to see what 
packets could be decoded by exhaustively searching through the data. In terms of run time, this algorithm took much longer. For instance, the mixed preclocking and original Goertzel preclocking took 3 minutes and 5 seconds and 2:36 respectively to run, while this exhaustive search took 26:48 on the 25:49 Track 1 of the test suite. This means that a 2.1Ghz Intel i7 (i7-3612QM) could not process the audio file in less time than elapses during the content of the audio file. With that being the case, the result is that with live data this approach would not work since it would continuously fall behind. Gratefully, this approach only decoded an additional 15 packets that the Correlation, original Goertzel (non-preclocking), and PLL did not decode. This result could be used to make the argument that the few more packets decoded is not worth the vast number more CPU cycles it takes to achieve it. 


\section{Results}

This results chapter is meant as a mechanism to present all of the data that was collected on the performance of different demodulators. All of the demodulators that were tested in the course of this research will be shown, whether it be dedicated hardware or software. The first results that will be shown are those of the dedicated hardware, being TNCs and Argent Data's OpenTrackers. Following the hardware will be the software implementations. Following these two categories will be some general comparison between them.

\subsection{Dedicated Hardware Results}

In the scope of this research a total of 12 pieces of hardware were tested. They include Argent Data's OpenTracker 2, OpenTracker 3, OpenTracker USB, OpenTracker 3 Micro, Kantronics KAM, two Kantronics KAM Plus, two AEA PK-88, a PK-232, a PK-232MBX, and an MFJ-1278. Hardware models for which more than one was tested will be differentiated by a number in parentheses following the model number. Please note that on the figures OpenTracker will be abbreviated OT.

The first two tests consisting of clean packets - 40 generated from the OpenTracker and 200 using Toledo's suite - was relatively uninteresting. Essentially every piece of hardware decoded all 40 and all 200 packets. The only anomalies to this were that the OpenTracker USB was only able to decode 39 and 193 out of the 40 and 200 packet files respectively. Additionally the OpenTracker 3 Micro missed one packet in the 200 packet file to only decode 199. Since there is no real way to debug and see the cause of decoding relatively fewer or more packets, just the data for these hardware items is presented as it was measured to allow for comparison to the software. This will continue to be the case for the remainder of this hardware section.

Following the two easy files the next file is same content as the file with 40 packets in it, with the only difference being that noise was progressively added. In Figure 9.1, the two PK-88s stand out for being able to decode 25 of the 40 packets in this file, which is a calculated $\mathrm{SNR}$ of 0.8. Just a reminder that in this figure and future figures that present results for this test file the number above each bar represents the caluclated SNR. 


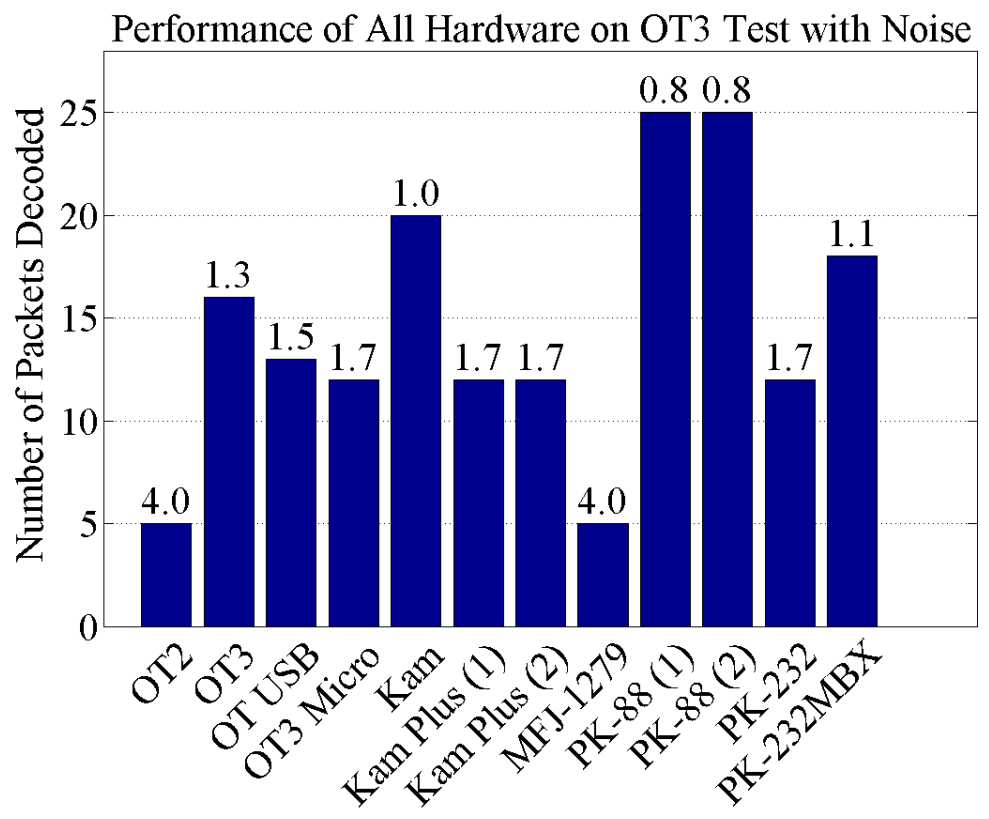

Figure 9.1. Number of packets successfully decoded for all tested hardware on the OpenTracker 3 test file with noise.

The next two files are the ones that were used most extensively in the testing for comparison and tuning. Primarily the first, which is just a recording of traffic off the air. They are Track 1 and 2 from the APRS CD mentioned in the Demodulator Benchmarking Chapter. The results from Track 1 are in Figure 9.2 and Track 2 in Figure 9.3. The top three performances of the hardware on Track 1 were the PK-88 (2) with 1007 packets decoded, the KAM with 988 Packets, and the KAM Plus (2) with 985 Packets. For Track 2 the top hardware was the KAM Plus (2) with 998, the KAM Plus (1) with 967, and the KAM with 938.

Using these results the best numbers for the hardware were 40 packets decoded from the Open Track 3 test, 200 from the javAX25 generated file, 25 from the Open Tracer 3 test with added noise, 1007 from Track 1 of the LA test suite, and 998 from Track 2. These best results can be used as a comparison for the software. 


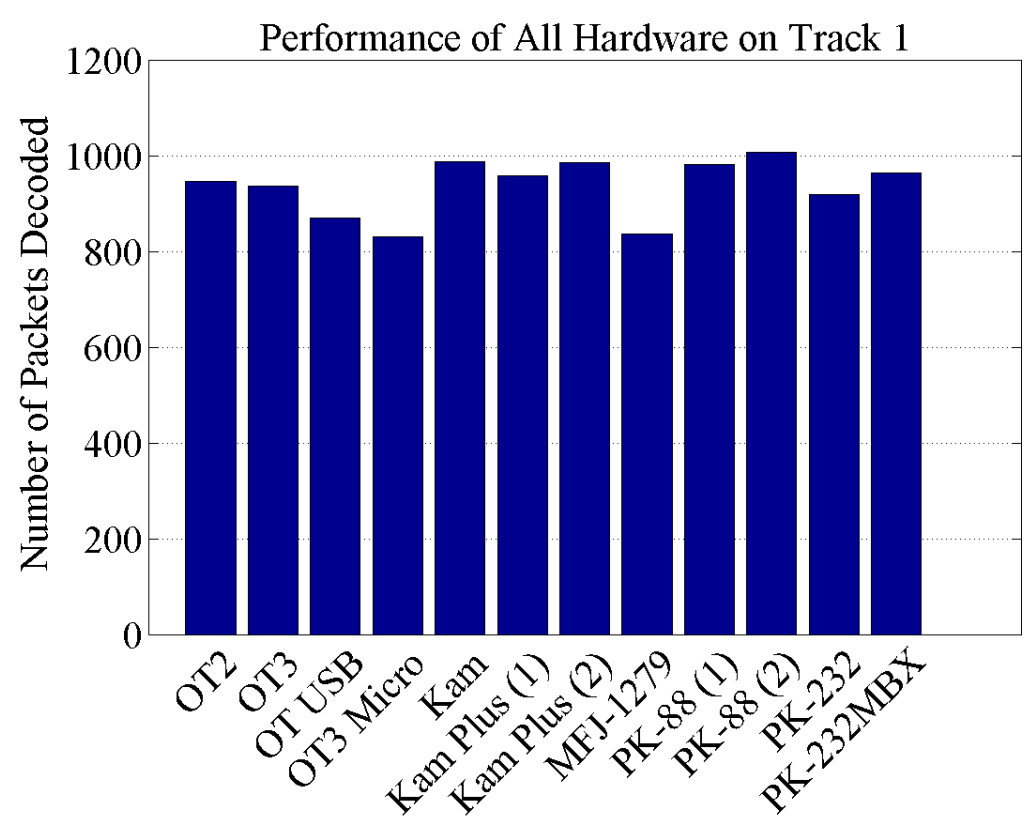

Figure 9.2. Number of packets successfully decoded for all tested hardware on the Track 1 test file.

\subsection{Software Results}

In this section, the number of packets that each of the demodulators in the javAX25 package was able to decode will be presented, highlighting those that were newly implemented in the course of this research. This will include the correlation approach that was already implemented before the start of this research as well as all of the new algorithms that were outlined in the previous chapter on Implementation. However, before getting the results from javAX25 there is one more data set to be introduced which is the results from another software based demodulation from AGW Packet Engine. Using this software, 40 packets were decoded from the OpenTracker 3 test, 200 from the javAX25 generated file, 21 from the OpenTracker 3 test with added noise (SNR of 1.1), 967 from Track 1 of the LA test suite, and 497 from Track 2. The results from javAX25 end up being on par with these results as well as those of the hardware.

With a total of 13 algorithms implemented, some did well and others not at all, so as in the section on hardware results, all of the data will be presented followed by a focus on 


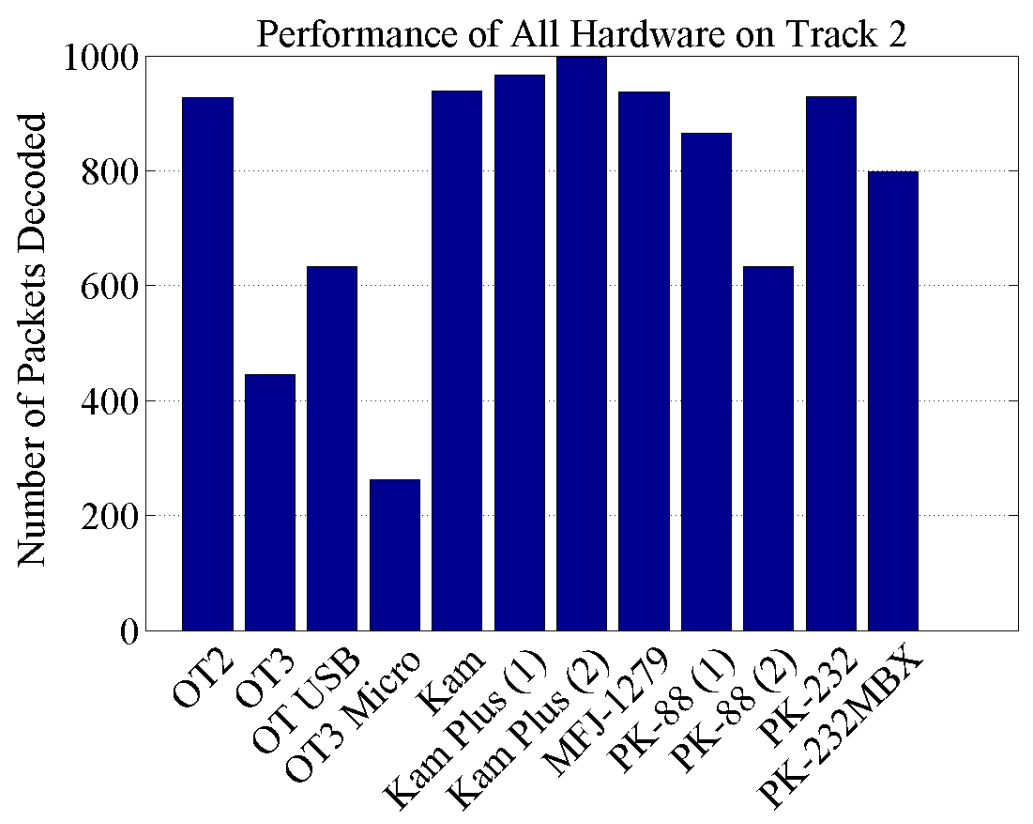

Figure 9.3. Number of packets successfully decoded for all tested hardware on the Track 2 test file.

those that performed the best. In the new javAX25 software implementation the filtering was moved from its original location of being on a per demodulator level basis to a central location that allowed each of the algorithms to utilize it. Some of the algorithms ended up relying on it after tuning and others could remain independent. As such, in order to present all of the data, each algorithm will not only have a result for each of the 5 test files, but also for each of the three filters used on the data. The three filters used are no filter, a $900-2500 \mathrm{~Hz}$ bandpass filter, and the same bandpass with a $6 \mathrm{~dB}$ attenuation of $1200 \mathrm{~Hz}$ tones to combat the signals that were not emphasized when transmitted but were deemphasized when received. For instance, for the Zero Crossing demodulator there will be three values for the OpenTracker 3 Test file, one at each filtering, and so on for the remaining 4 test files.

As correlation was the original implementation and already present in the javAX25 package its performance should be considered the baseline. Since this is the baseline it will be on the far left of the plots for reference. The performance of no filter on the OpenTracker 3 Test can be seen in Figure 9.4, Figure 9.5 shows data with the bandpass filter, and the 


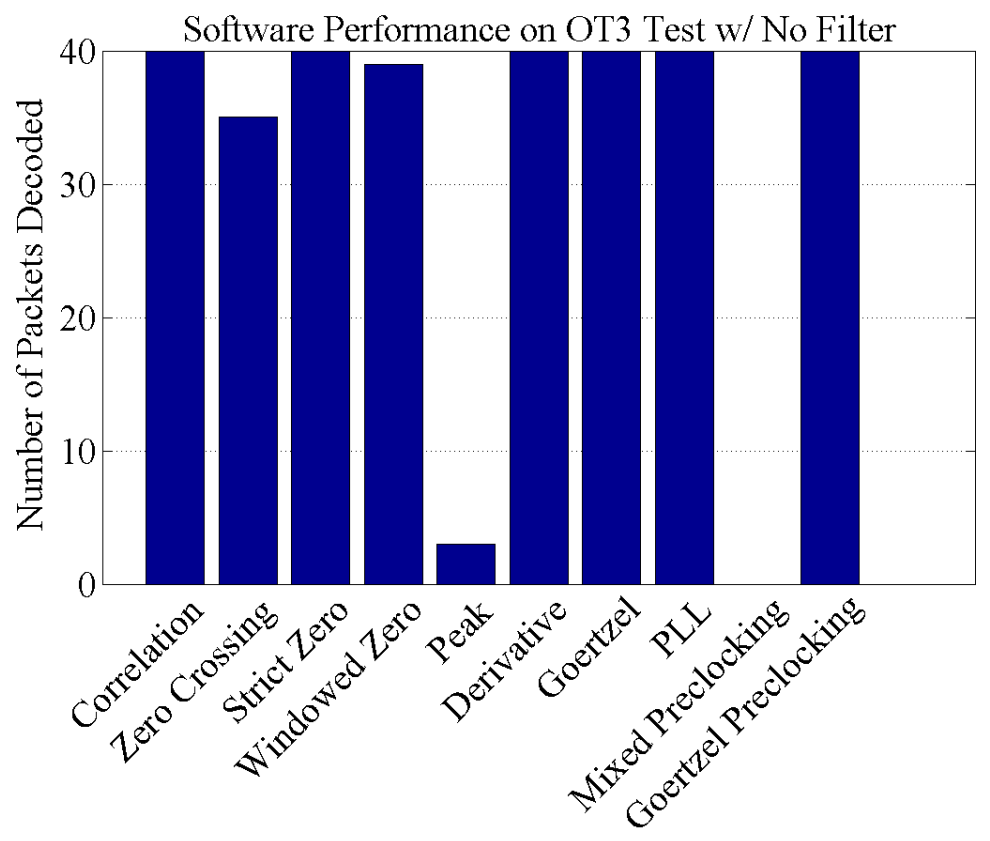

Figure 9.4. Performance of software on the raw signal from OpenTracker 3 Test.

emphasizing filter results in Figure 9.6. It can be observed that zero crossing did not favor the filters, others were resilient, and preclocking thrived. The Generated 200 Test file is the only file that the peak demodulator performed comparably to the other techniques. The good performance of the peak demodulator on only this file was due to the fact that it was tuned using this file. As it was attempted to optimize it for other files, it was found that the peak demodulation was too delicate which has a lot to do with its poor performance on the other files. The unfiltered data from Generated 200 test is in Figure 9.7, the bandpass data in Figure 9.8, and the results of emphasizing the signal in Figure 9.9. Although the generated 200 packet file and the OpenTracker test file had similar results, the results for the generated 200 were better. This can be attributed to the fact that this file has only ever existed in the digital realm, it was made and consumed there, as opposed to being produced in the physical world and then recording and digitizing it.

Again, moving on from the "easy" files the performance of the software demodulators on the OpenTracker 3 Test with the noise added can be seen. Figure 9.10 shows the data for the unfiltered file, Figure 9.11 shows bandpass filter data, and Figure 9.12 shows data from the emphasis filter. Two algorithms really start to shine as being comparable, and in some 


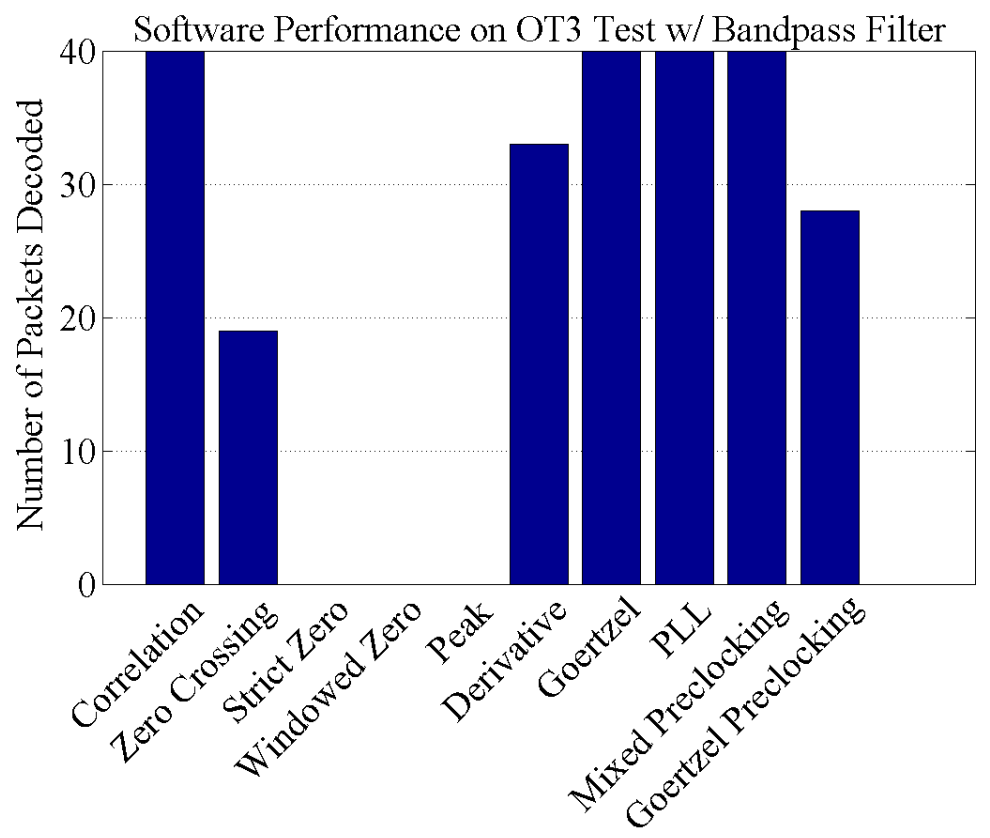

Figure 9.5. Performance of software on OpenTracker 3 Test with a flat bandpass filter.

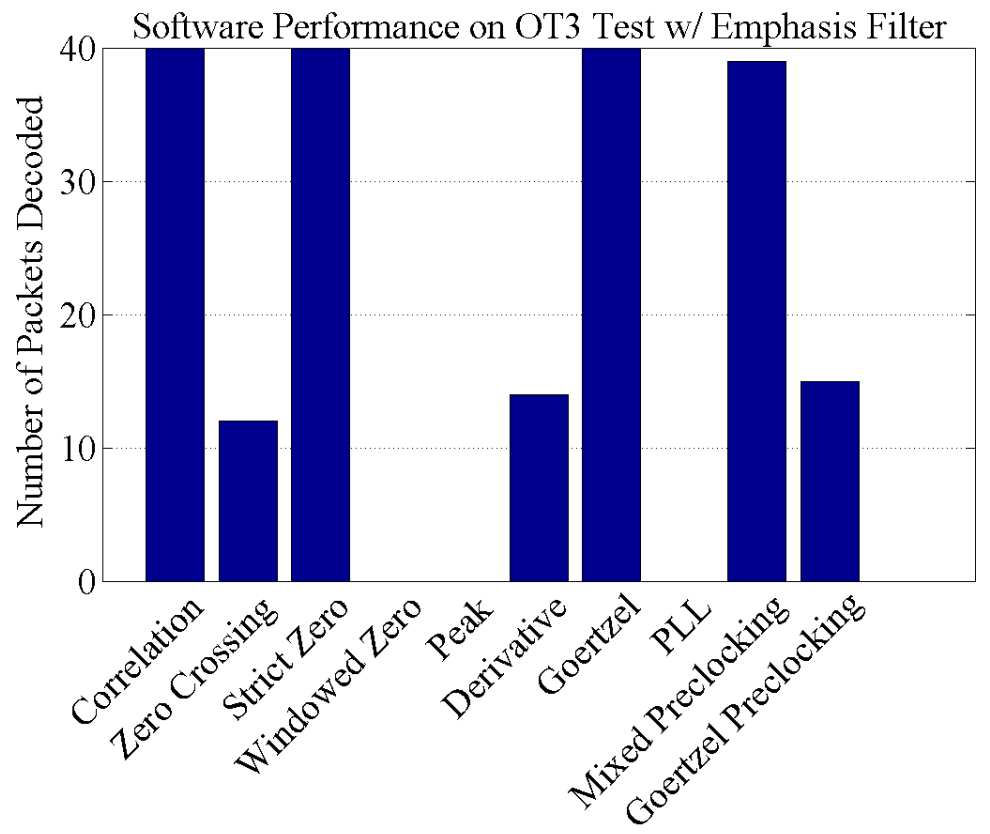

Figure 9.6. Performance of software on OpenTracker 3 Test with an emphasis filter. 


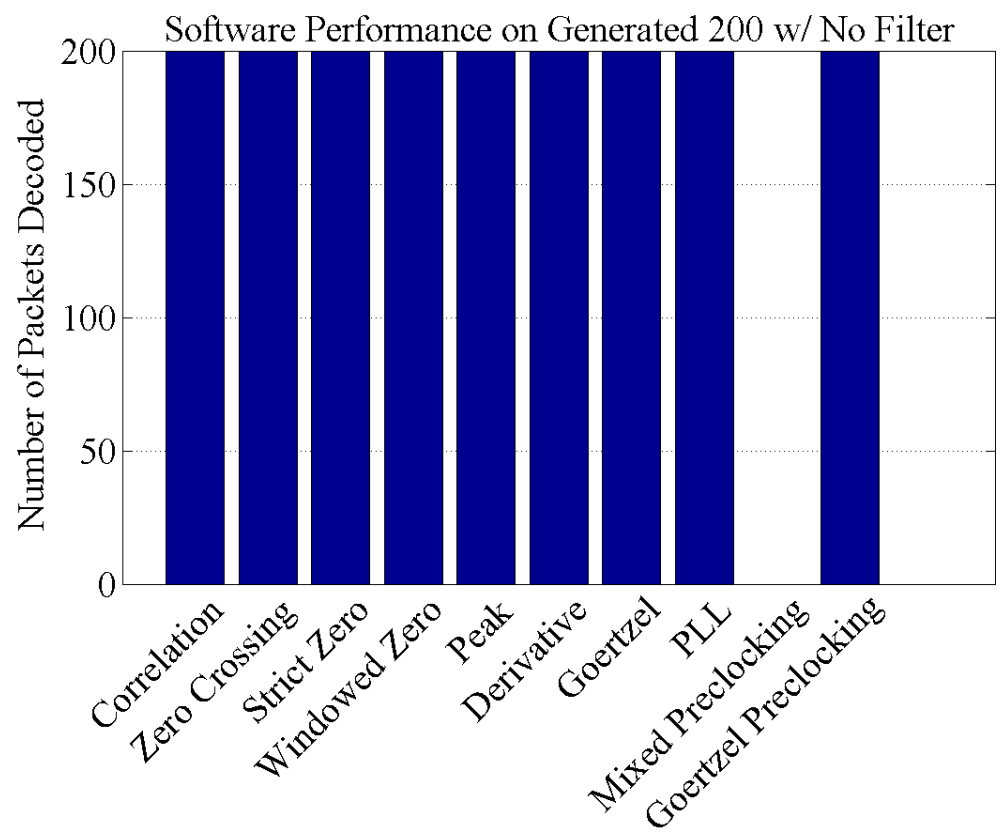

Figure 9.7. Performance of Software on the raw signal from Generated 200.

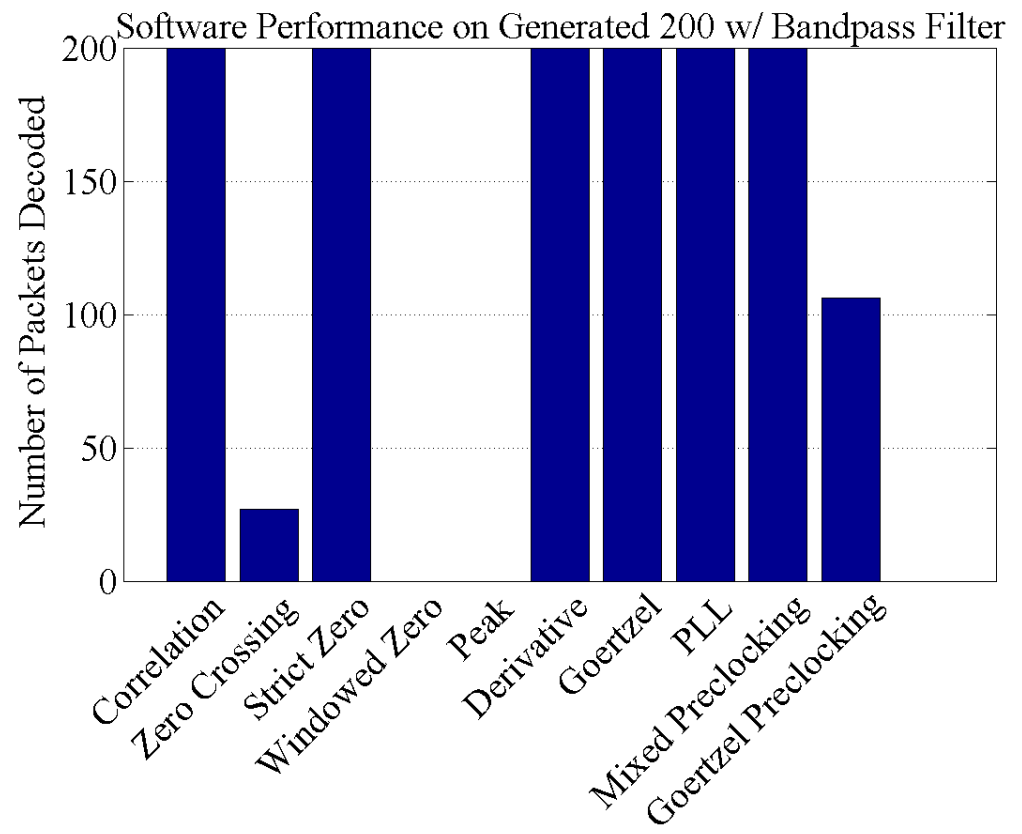

Figure 9.8. Performance of software on Generated 200 with a flat bandpass filter. 


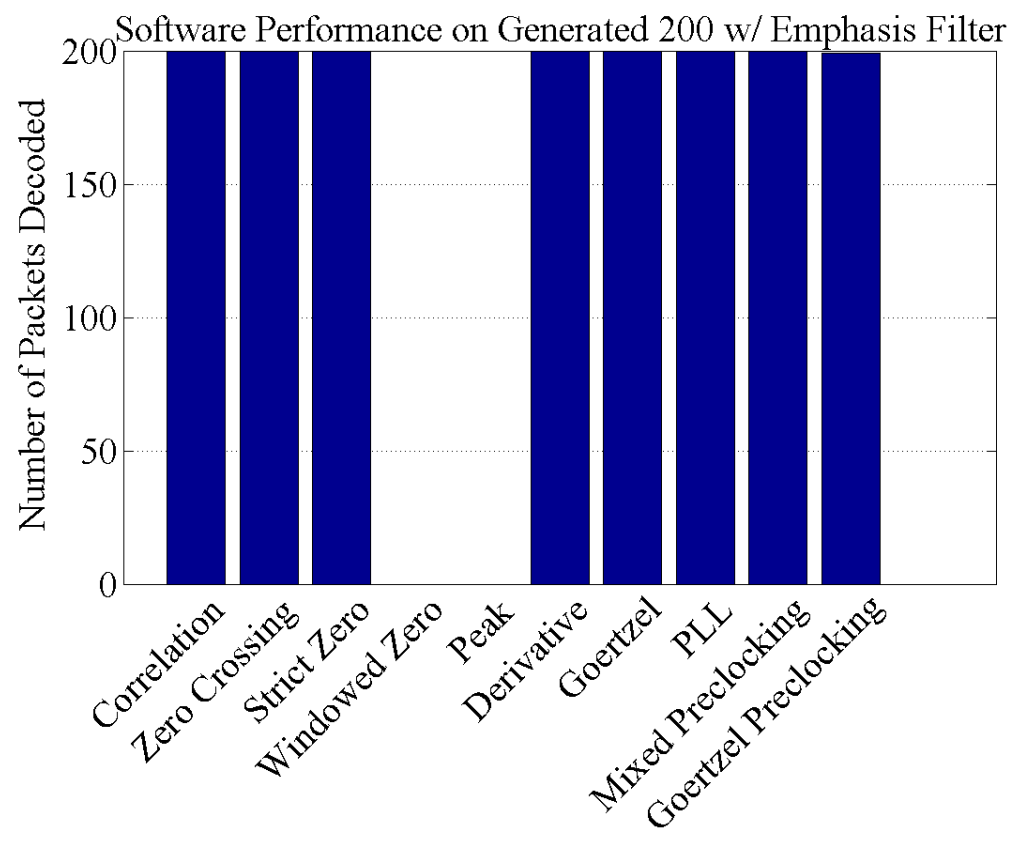

Figure 9.9. Performance of Software on Generated 200 with an emphasis filter.

cases better, than the correlation demodulator. Those are the Goertzel Filter Demodulator and the PLL Demodulator. Although this is not data that was actually transmitted or received, these two start to show promise. Even the Strict Zero crossing can be seen doing well once the audio file is emphasized, but it doesn't stand up to the competition in the next two test files containing off air data.

As mentioned earlier, these next two test files were those that were thought the be most important to succeed at. As such, Track 1 was used to tune the algorithms since this would represent the closest real-world simulation possible. The Track 2 results are also presented for comparison. However in Track 2, in addition to being deemphasized the process of this filtering also reduced the magnitude of the signal in the audio file as mentioned earlier. As such, this file shows two things: One, the ability to pick up lower level signal and second, the algorithms tolerance to signals that were not emphasized when transmitted, but were deemphasized when received. The results of no filtering, bandpass filtering, and emphasis filtering can be seen in their respective Figure 9.13, 9.14, and 9.15 for Track 1. The data from demodulating Track 2 can be seen in Figure 9.16, 9.17, and 9.18. As was caught during the OpenTracker 3 Test with noise it can be noticed that the Goertzel and PLL 


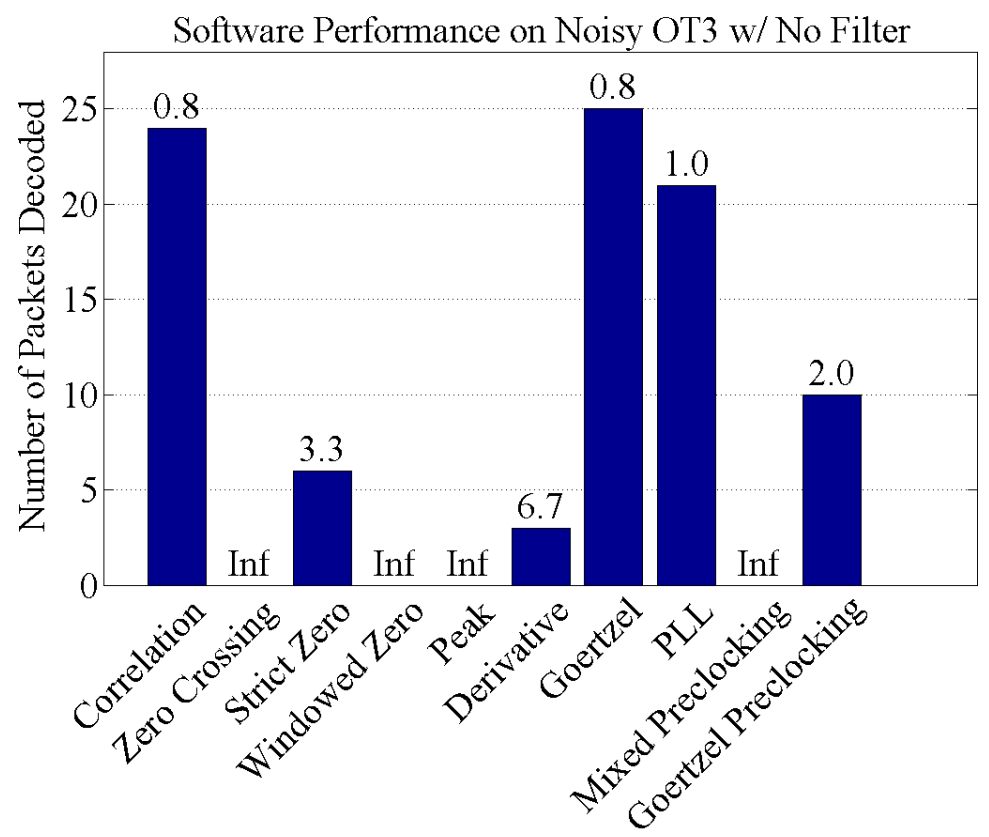

Figure 9.10. Performance of software on the raw signal from OpenTracker Test with noise added.

algorithms are still doing well on Track 1, and also the Mixed preclocking is doing fairly well. With the filter that is applied on Track 1 to create Track 2 it is basically the opposite effect of Toledo's emphasis filter and hence they cancel each other out. This can be seen by looking at the performance of the Goertzel Demodulator which does poorly with both other filterings on Track 2. However, its ability to detect low level signals and this reversal of the emphasis filtering applied makes it end of having comparable results to just the band pass filter on Track 1 - 956 versus 965 .

\subsubsection{Hardware and Software Comparisons}

It is now time to actually compare the new software implementations to the old ones as well as to the hardware. The first thing to notice is that the Goertzel Filter did very well, and in some cases better than the original correlation. Secondly, the complicated preclocking algorithm was also able to hold its own and still be a top contender. In fact the top three software algorithms were Correlation, Goertzel, and Mixed Preclocking with 964, 965, and 


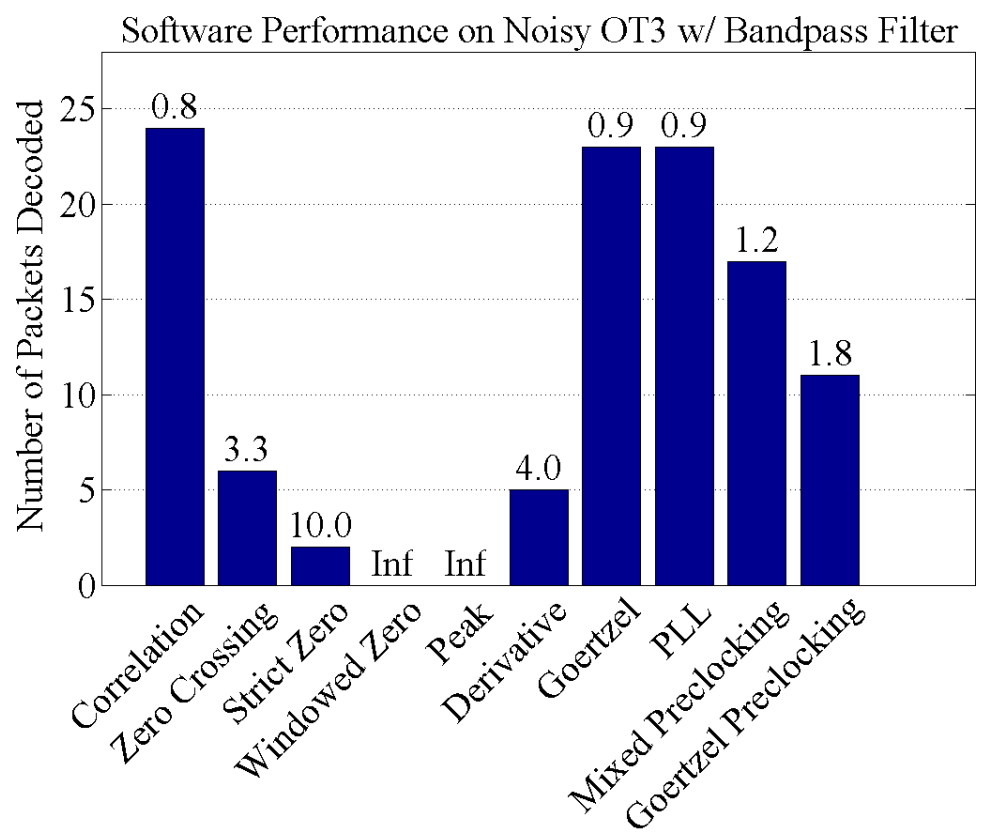

Figure 9.11. Performance of software on OpenTracker Test with noise added with a flat bandpass filter.

939 packets decoded from Track 1 with the bandpass filter, the results of these top three algorithms versus the top three pieces of hardware can be seen in Figure 9.19. In addition to having the top 3 of each Figure 9.19 includes the results of AGWPE and running the top 3 software algorithms in parallel. Even though each algorithm decodes different numbers of packets they each still have their expertise with being able to exclusively decode packets that others could not. For instance when the three are run together there is a total of 975 packets decoded due to the fact that Goertzel gets 12 packets that the other two do not, Correlation gets 7 that the others do not, and Preclocking decoded 3 that these other two missed. This could still be a good argument for the use of software over hardware since it is very easy to run multiple demodulators in parallel. Especially since the cost of this is only 5 minutes and 2 seconds on a file that is 25:49 long.

The question is, is the software better than the hardware? Looking at the highest numbers, no, but looking at the bigger picture, maybe. One thing about the hardware is that it is prone to variations and in need of periodic tuning. So, if instead of looking at the best of the breed for the hardware, if the average values are compared under the presumption that 


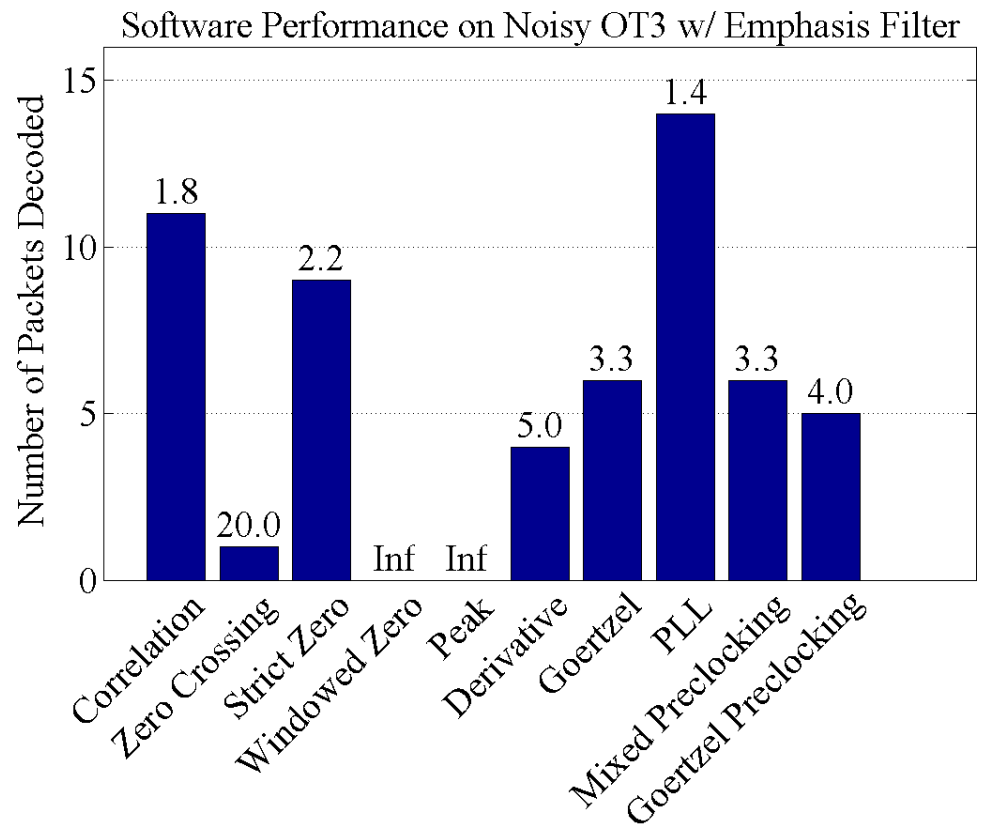

Figure 9.12. Performance of Software on OpenTracker Test with noise added with an emphasis filter.

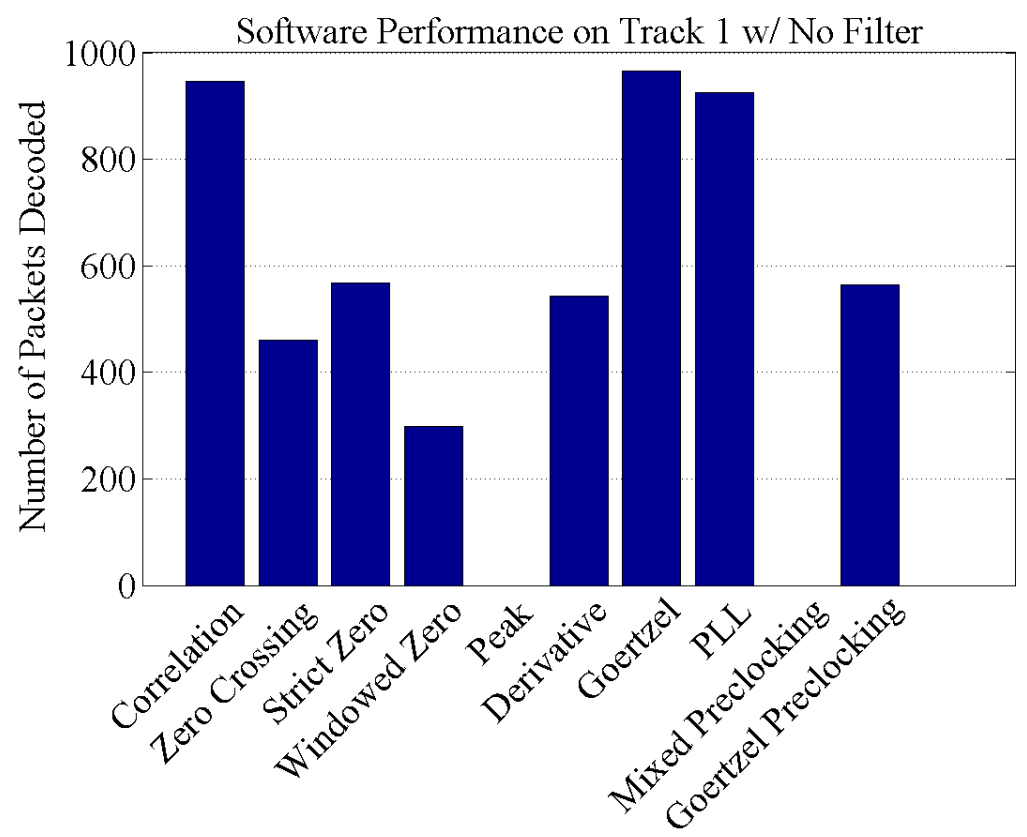

Figure 9.13. Performance of software on the raw signal from Track 1. 


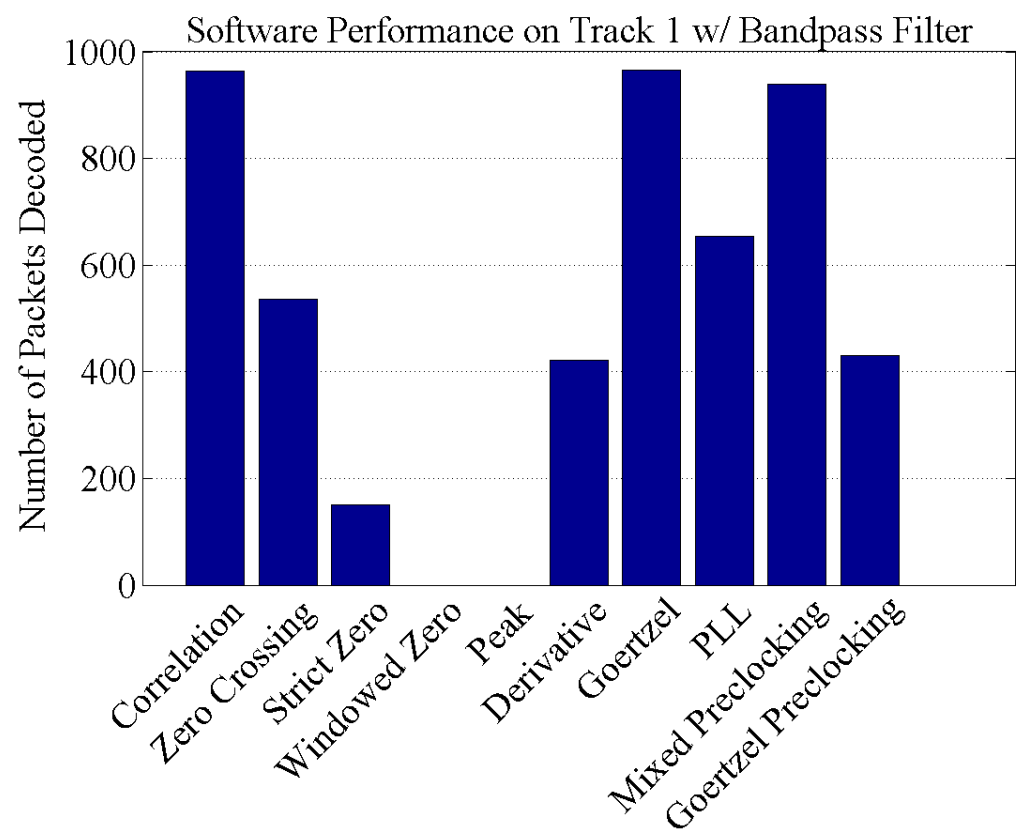

Figure 9.14. Performance of software on Track 1 with a flat bandpass filter.

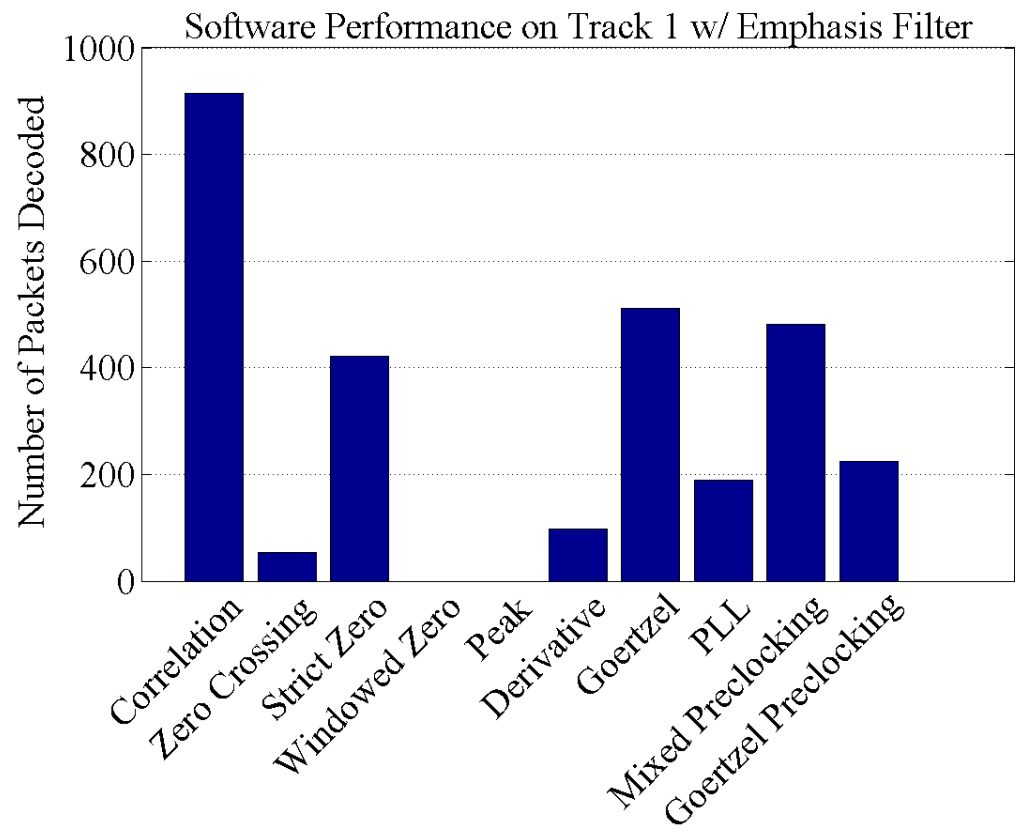

Figure 9.15. Performance of software on Track 1 with an emphasis filter. 


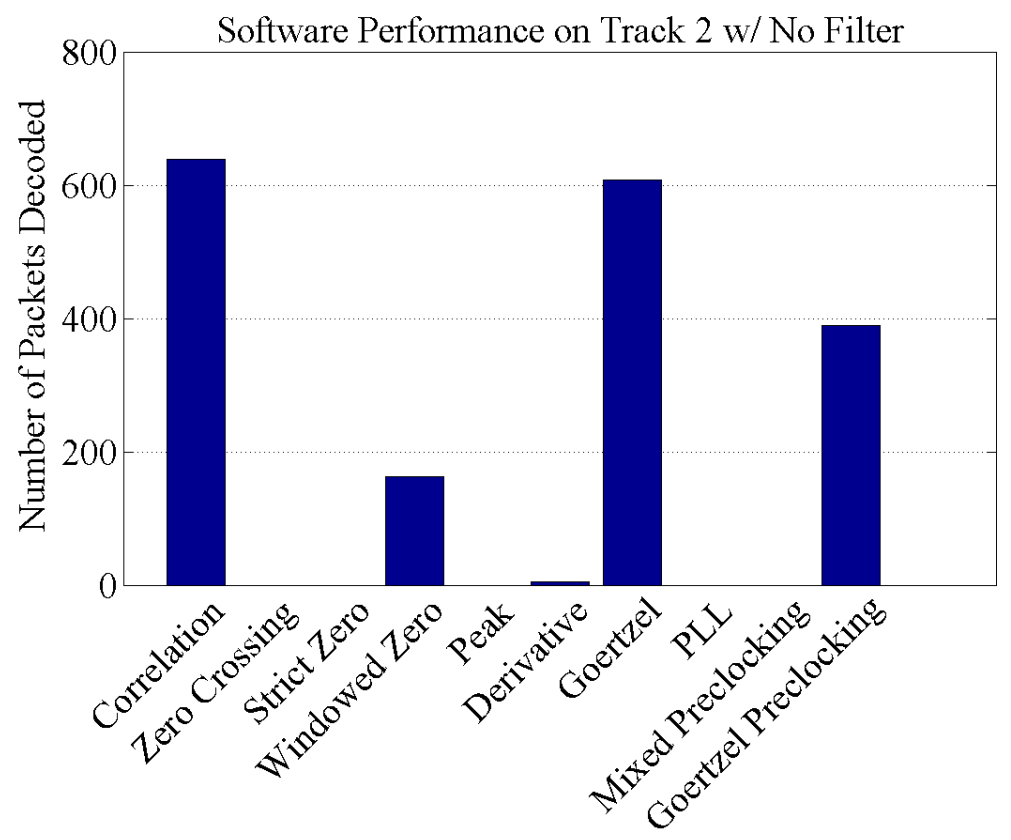

Figure 9.16. Performance of software on the raw signal from Track 2.

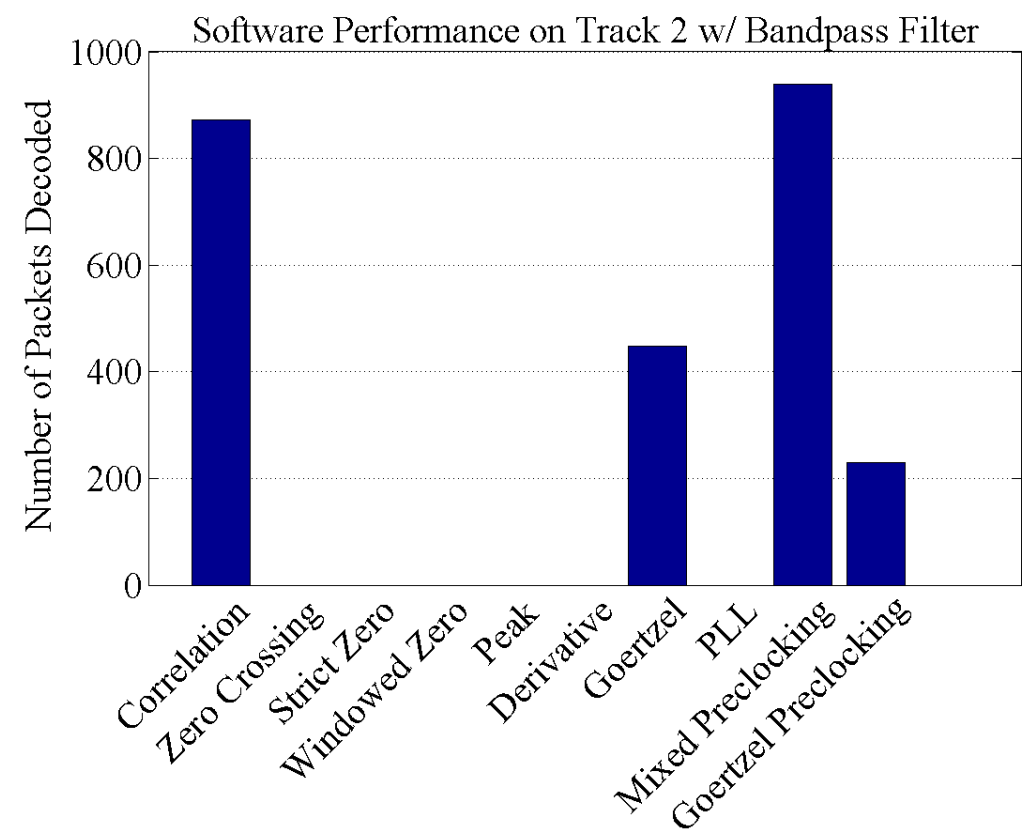

Figure 9.17. Performance of software on Track 2 with a flat bandpass filter. 


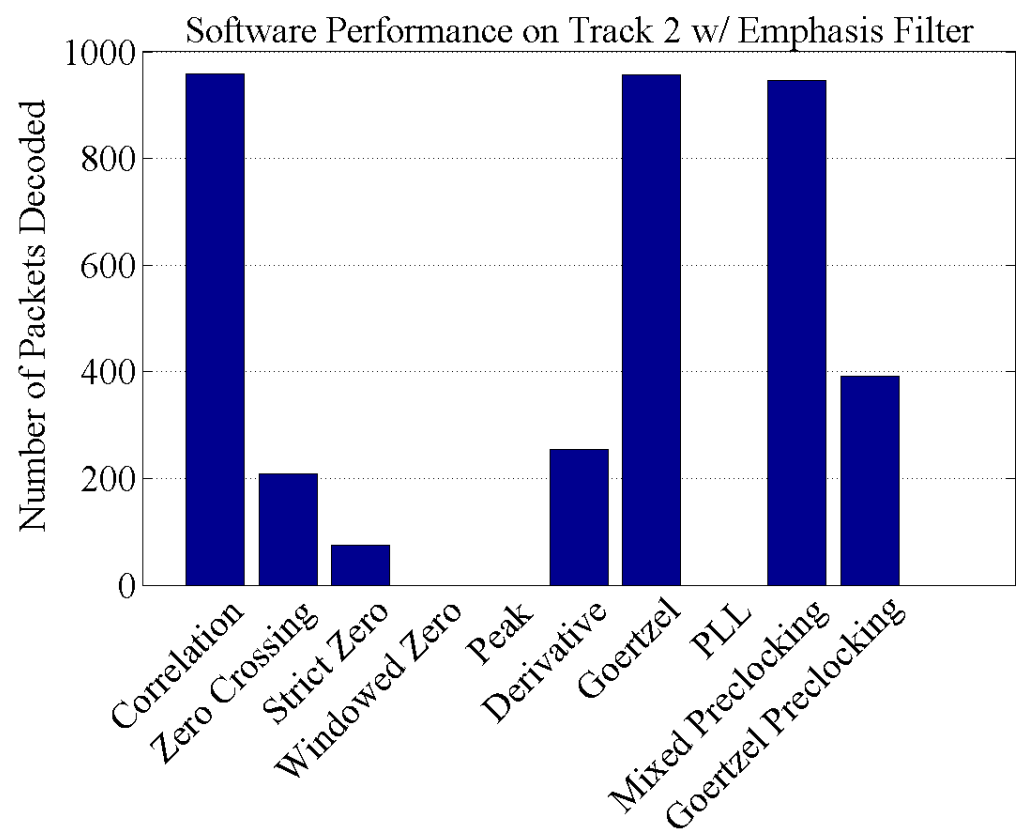

Figure 9.18. Performance of software on Track 2 with an emphasis filter.

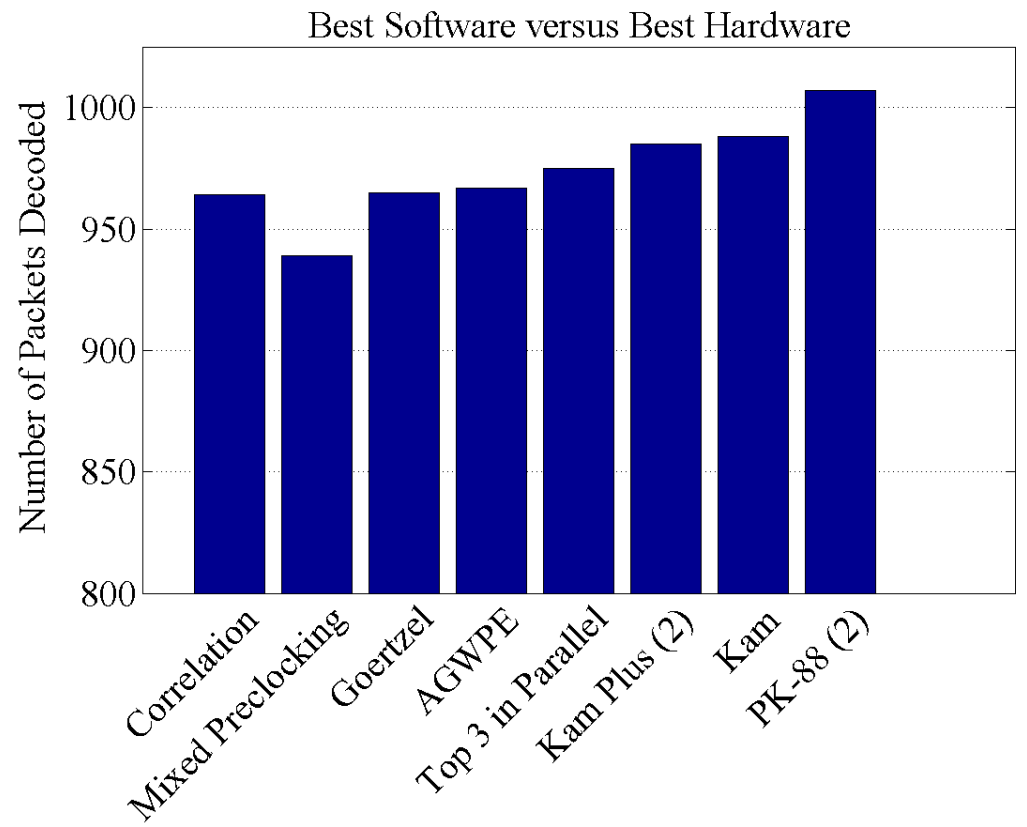

Figure 9.19. Performance of software versus hardware on Track 1. 
this would correspond to the average ham's decoding capabilities, then the software does decode more packets. Additionally, the software does not have any capacitors that will dry up or solder joints that may become brittle and affect the performance. The performance of the software today will be exactly the same in 5, 10, many years. The average number of packets decoded by the hardware on Track 1 was 935 . Looking at this value any one of the three algorithms that are the top performers would be considered better. 


\section{Future Work}

The sections in this chapter will explain some aspects that this project uncovered that have the potential for future research. There are three main items that the author thinks should be explored in more depth as future work in this area of AX.25 software based demodulation, including the Discrete Short-Time Fourier Transform, the use of the checksum for forward error correction, and to actually integrate these demodulators with live traffic from a radio.

\subsection{The Discrete Short-Time Fourier Transform}

In a paper by Zhonghui-Chen et. al., methods are outlined to use the discrete short-time Fourier transform (DSTFT) to demodulate a binary frequency shift keyed (BFSK) signal. After a discussion of the DSTFT they go into their specific implementation, but this appears to show promise because of their results section. Granted, this was for a simulation but they showed that the error rate was lower using the DSTFT than traditional coherent demodulation even for lower signal to noise ratios [11].

\subsection{Use the CRC for FEC}

Each AX.25 packet contains a checksum that is generated by using a cyclic redundancy check (CRC). This is used by all demodulators in order to determine if the packet that the demodulator thinks it decoded was actually a legitimate packet or just noise that happened to look like a packet. Although the CRC was not intended for forward error correction (FEC), it would be interesting to see the effects of using it as such. With algorithms such as the preclocking algorithms, the power of each symbol is determined and this power could be used to assign a level of confidence on that demodulated bit. If a packet fails the CRC check, and all except for one of the bits has a confidence greater than 80 percent, would the CRC pass if that one bit was flipped to the other symbol? This is a very good argument for the use of software since this meta data about the decoding of the packets can be kept in memory, something that most hardware does not have much of. 


\subsection{Integrations with a Radio}

Finally another area for future work would be to integrate the new algorithms with a radio and use them to decode live data. Although the data used in the testing was a recording of live data, it would be very gratifying to be able to see these algorithms decode audio straight from the radio. Inside of javAX25, the packages should already support it, so it should be a matter of just setting it up and letting it run. This analysis would allow for verification of the implementations' functionalities as well as to be able to see how the different algorithms perform side by side in real time. 


\section{Conclusion}

This research set out to try and prove that software could do APRS demodulation better than the hardware. Depending on perspective, it may have done so. However, at the very least the research shows that there are many ways to approach the challenge of demodulating these packets and presents the relative performance of over 10 software based approaches and 12 dedicated hardware approaches, whether that hardware is an OpenTracker or TNC.

In the end, the software did do better than the average result using hardware, but in the primary benchmarking file, software was only able to decode 975 packets as opposed to the 1007 that the best piece of hardware detected. The software's improved performance coupled with its low cost, continues to make it an attractive approach over dedicated hardware. Exploring some of the items in the future work section such as using the checksum for forward error correction may be able to get software to outperform hardware. 


\section{BIBLIOGRAPHY}

[1] 600/1200-baud modem standarized for use in the general switched telephone network. https://www.itu.int/rec/T-REC-V.23-198811-I/en, November 1988. V.23.

[2] Inc. Advanced Electronic Applications. Model pk-88 packet conroller operating manual. http://www.repeater-builder.com/aea/pdf/aea-pk-88-user-manual. pdf, 1990.

[3] Salam Akoum and Behrouz Farhang-Boroujeny. A phase locked lopp with arbitrarily wide lock range for software defined radios. http://www.ece.utah.edu/ akoum/ PLL_Paper.pdf.

[4] William A. Beech. Ax.25 link access protocol for amateur packet radio. https://www . tapr.org/pdf/AX25.2.2.pdf, July 1998.

[5] Carl Bergquist. Ham Radio Operator's Guide. PROMPT Publications, second edition edition, 2001.

[6] Bob Bruninga. Automated packet reporting system. http://aprs.org/.

[7] Bob Bruninga. Aprs spec addendum 1.1. http://www.aprs.org/aprs11.html, July 2004.

[8] Bob Bruninga. Aprs spec addendum 1.2 proposals. http://www.aprs.org/aprs12. html, July 2013.

[9] Byonics. Byonics tinytrak. http://www. byonics.com/tinytrak/.

[10] Robert Campbell. javax25 (github). https://github.com/robbieford/javAX25, 2015.

[11] Zhonghui Chen, Haibin Lin, Xin Chen, and Huiqun Hong. Fsk signal demodulation method based on dstft. In Wireless Communications, Networking and Mobile Computing, 2008. WiCOM '08. 4th International Conference on, pages 1-3, Oct 2008.

[12] APRS Community. Aprs-is. http://www.aprs-is.net/, 2015. 


\section{BIBLIOGRAPHY}

[13] Kenwood U.S.A Corporation. Tm-d700a owner manual. http://www . kenwoodusa.com/UserFiles/File/UnitedStates/Communications/AMA/ Manuals/TM-D700-Owner-Manual.PDF.

[14] J. Das, S. K. Mullick, and P. K. Chatterjee. Principles Of Digital Communication. John Wiley \& Sons, 1986.

[15] Argent Data. Ot3m case front. http://www.argentdata.com/catalog/images/ Ot3m-termblk.jpg.

[16] Advanced Micro Devices. Am7910/11 fsk modem datasheet. http://pdf1. alldatasheet.com/datasheet-pdf/view/124524/AMD/AM7910.html, June 1989.

[17] Wilfried Elmenreich. Emodulation detecting a frequency using a goertzel filter. http: //netwerkt.wordpress.com/2011/08/25/goertzel-filter/, August 2011.

[18] EXAR. Xr-2211a data sheet. https://www.exar.com/common/content/document. ashx?id=170, June 1997.

[19] Kenneth W. Finnegan. Examing ambiguities in the automated packet reporting system. Master's thesis, California Polytechnic State University San Luis Obispo, 2014 .

[20] Kenneth W. Finnegan and Bridget Benson. Clarifying the amateur bell 202 modem. 33rd ARRL and TAPR Digital Communications Conference, 2014.

[21] Joseph D. Gaeddert. Wrloops a phase-locked loop in straight c. http://liquidsdr. org/blog/pll-howto/, November 2013.

[22] Michaek D. Gallagher and Randall A. Snyder. Mobile Telecommunications Networking With IS-41. McGraw-Hill, 1997.

[23] Stan Gibilisco, editor. Amateur Radio Encyclopedia. TAB Books, 1994.

[24] Lillian Goleniewski. Telecommunications Essentials. Addison-Wesley, second edition edition, 2006.

[25] Darien Graham-Smith. How to: How much ram do you re- 


\section{BIBLIOGRAPHY}

ally need? http://www.pcauthority.com.au/Feature/375815, how-to-how-much-ram-do--you-really-need.aspx, March 2014.

[26] GTA-West Regional Packet Group. Introduction to packet radio for ares. http:// barc.ca/wordpress/wp-content/uploads/2014/07/Packet_Basics_GTAW.pdf, 2012 .

[27] The APRS Working Group. Automatic position reporting system: Aprs protocol reference. http://www. aprs.org/doc/APRS101.PDF, August 2000.

[28] Harry Helms. All About Ham Radio. HighText Publications, 1992.

[29] Wayne Holder. Wayne's tinkering page: Bell 202, 1200 baud demodulator in an attiny10. https://sites.google.com/site/ wayneholder/attiny-4-5-9-10-assembly-ide-and-programmer/ bell-202-1200-baud-demodulator-in-an-attiny10, July 2012.

[30] Stan Horzepa. Your Gateway to Packet Radio. The American Radio Relay League, 1992.

[31] Timewave Technology Inc. Pk-232 mbx operating manual. http://www . repeater-builder.com/aea/pk232/pk232mbx-operating-manual .pdf, 2001.

[32] National Instruments. Keying and digital modulation. http://www.ni.com/ white-paper/3013/en/, November 2014.

[33] Texas Instruments. Tcm 3105 fsk modem datasheet. http://www.netti.fi/ ryydis/ tcm3105.pdf, May 1994.

[34] Javvin. Network Protocols Handbook. Javvin Technologies, Inc., third edition edition, 2006.

[35] Kantronics. Kam plus getting started. http://www.ocrg.org/documents/Kam\% 20Plus\%20Getting\%20Started.pdf, 1993.

[36] Kantronics. Kpc-3+ packet communicator. http://www.kantronics.com/products/ kpc3.html, 2014. 


\section{BIBLIOGRAPHY}

[37] KK6RF. Kantronics kam plus v8.2 firmware and 512kb. http://forums.qrz.com/ attachment $\cdot$ php?attachmentid=192170\&d=1406602474\&thumb=1.

[38] Georg Lukas. Aprsdroid (github). https://github.com/ge0rg/aprsdroid, December 2013.

[39] Paul Lutus. Understanding phase-locked loops. http://arachnoid.com/phase_ locked_loop/, 2011.

[40] Edward Claro Mader. What is the speed of electricity and why? https://www .quora. com/What-is-the-speed-of-electricity-and-why, July 2014.

[41] Bruce W. Martin. Radio, tnc \& gps pinouts. http://www.bwm.us/Resource/radio_ pinout.html, June 2014.

[42] Dominic Mazzoni. Audacity. http://audacity.sourceforge.net/.

[43] MFJ. Model mfj-1278/mfj-1278t instruction namual. http://www.radiomanual. info/schemi/ACC_packet/MFJ-1278_MFJ-1278T_user.pdf, 1990.

[44] Scott Miller. Aprs and packet radio products. http://www.argentdata.com/ products/aprs.html.

[45] James A. Mitrenga. An mx614 packet modem. http://www.cmlmicro.com/assets/ 614_TCM3105.pdf, January 2000.

[46] Newegg. Rosewill rc-701 5.1 channels pci interface sound card. http://www. newegg. com/Product/Product . aspx?Item=N82E16829265001, 2014.

[47] Ham Radio Outlet. Kantronics kpc-3 plus. http://www.hamradio.com/detail.cfm? pid=HO-000229, 2014.

[48] Michael H. Perrott. Tutradio on digital phase-locked loops. http://www.cppsim.com/ PLL_Lectures/digital_pll_cicc_tutorial_perrott.pdf, September 2009.

[49] Larry L. Peterson and Bruce S. Davie. Computer Networks: A Systems Approach. Elsevier, fifth edition edition, 2011.

[50] John G. Proakis. Digital Communications. McGraw-Hill Book Company, 1983. 


\section{BIBLIOGRAPHY}

[51] Thaddeus A. Roppel. Fsk-frequency shift keying. http://www.eng.auburn . edu/ troppel/courses/TIMS-manuals-r5/TIMS\%20Experiment\%20Manuals/ Student_Text/Vol-D1/D1-07.pdf.

[52] George Rossopoylos. Agw packet engine. http://www.sv2agw.com/ham/agwpe.htm.

[53] David Rowe. Fsk over fm. http://www.rowetel.com/blog/?p=3799, December 2014.

[54] Thomas Sailer. Internationale packet-radio tagung darmastadt: Dsp modems. http://web.archive.org/web/*/http://www. baycom.org/ tom/ham/da95/d_ dspmod.pdf, 1995.

[55] Thomas Sailer. Using a pc and a soundcard for popular amateur digital modes. http: //www. tapr.org/pdf/DCC1997-Soundcard4Digital-HB9JNX.pdf, 1997.

[56] Thomas Sailer. Soundmodem on modern operating systems. https://www.tapr .org/ pdf/DCC2000-SOUNDMODEMonOS-HB9JNX-AE4WA .pdf, August 2000.

[57] Mitra Sanjit K and James F. Kaiser, editors. Handbook For Digital Signal Processing. John Wiley \& Sons, 1993.

[58] Dennis Seguine. Simplified fsk signal detection. http://dx.eng.uiowa.edu/ eedesign/fskcorr.pdf, September 2006.

[59] Cypress Semiconductor. Modulation and demodulation. http://www.cypress.com/ ?docID $=43670$.

[60] H. Ward Silver, editor. The ARRL Handbook for Radio Communications 2014. The Amatuer Radio Relay League, Inc., 91 edition, 2013.

[61] Marvin K. Simon, Sami M. Hinedi, and William C. Lindsey. Digital Communication Techniques. PTR Prentice Hall, 1995.

[62] Bernard Sklar. Digital Communications Fundamentals and Applications. Prentice Hall, 1988.

[63] Stephen H. Smith. Tnc test cd. http://www.wa8lmf .net/TNCtest/, August 2009. 


\section{BIBLIOGRAPHY}

[64] Stephen H. Smith. Automatic position reporting system. http://wa8lmf .net/aprs/, January 2012.

[65] 4Gon Solutions. Factors affecting wireless networking performance. http: //www.4gon.co.uk/solutions/technical_factors_affecting_wireless_ performance.php\#range.

[66] Barrie Sosinsky. Networking Bible. Wiley Publishing, Inc., 2009.

[67] M.K. Stauffer. Fsk voiceband modem using digital filters. http://www.google.com/ patents/US4425665, January 1984. US Patent 4,425,665.

[68] Argent Data Systems. Argent data systems opentracker usb users manual. https: //www.argentdata.com/support/OpenTrackerUSB_manual.pdf, February 2013.

[69] Fox Delta Project Team. Foxtrak :: Aprs viewers, trackers \& gps encoder. http: //www.foxdelta.com/products/foxtrak.htm.

[70] Sivan Toledo. Ax25 java soundcard modem. https://github.com/sivantoledo/ javAX25/blob/master/manual.doc, February 2012.

[71] Sivan Toledo. A high-performance sound-card ax.25 modem. QEX, July / August 2012:19-25, 2012.

[72] Sivan Toledo. javax25 (github). https://github.com/sivantoledo/javAX25, April 2012.

[73] Yaesu USA. Ftm-350 series aprs manual. http://www.yaesu.com/ indexVS. $c f m ? c m d=D i s p l a y P r o d u c t s \& P r o d C a t I D=106 \&$ encProdID $=$ 33C814E3D04C92310507ECDE68CC3C01\&DivisionID.

[74] Bob Watson. Fsk: Signal and demodulation. WJ Communication, 7, 1980.

[75] APRS Wiki. Digipeater. http://info.aprs.net/index.php?title=Digipeater, November 2012.

[76] Wikipedia. Automated packet reporting system. https://en.wikipedia.org/wiki/ Automatic_Packet_Reporting_System. 


\section{BIBLIOGRAPHY}

[77] Wikipedia. Continuous-phase frequency shift keying. http://en.wikipedia.org/ wiki/Continuous_phase_modulation\#Continuous-phase_frequency-shift_ keying, March 2014.

[78] Wikipedia. Discrete forier transform. http://en.wikipedia.org/wiki/Discrete_ Fourier_transform, November 2014.

[79] Wikipedia. Fast forier transform. http://en.wikipedia.org/wiki/Fast_Fourier_ transform, November 2014.

[80] Wikipedia. Fourier transform. http://en.wikipedia.org/wiki/Fourier_ transform, November 2014.

[81] Wikipedia. Goertzel algorithm. http://en.wikipedia.org/wiki/Goertzel_ algorithm, November 2014.

[82] Larry D. Wolfgang, editor. Now You're Talking! All you Need for Your First Amateur Radio License. The Amatuer Radio Relay League, Inc., 5th edition edition, 2005.

[83] Yaesu. Ftm-350 high res. http://www yaesu.com/downloadFile.cfm?FileID=5183\& FileCatID=171\&FileName=FTM\%2D350US\%5F . jpg\&FileContentType=image \% 2Fpjpeg. 